\title{
Boundary geometric control of a counter-current heat exchanger
}

\author{
Ahmed MAIDI $^{a}$, Moussa DIAF ${ }^{b}$, Jean-Pierre CORRIOU ${ }^{a}$ * \\ ${ }^{a}$ Laboratoire des Sciences de Génie Chimique, CNRS-ENSIC, \\ 1, Rue Grandville BP 20451, 54001 Nancy Cedex, France. \\ ${ }^{b}$ Université Mouloud MAMMERI, \\ Faculté de Génie Electrique et d'Informatique, \\ Département Automatique, 15000 Tizi-Ouzou, Algérie.
}

\begin{abstract}
The present article deals with the boundary geometric control of a counter-current heat exchanger whose control is designed considering a model based on two partial derivative equations describing the variations of internal and external temperatures. The objective consists in controlling the internal fluid temperature, at the heat exchanger outlet, by manipulating the jacket temperature at its inlet boundary in spite of the variation of the temperature of the internal fluid at the heat exchanger inlet. The control law is designed considering the partial differential equation describing the temperature of the internal fluid, and the manipulated control is the boundary condition for the partial differential equation describing the temperature of the jacket fluid. The performances of the controller have been evaluated by simulation and the results show that it provides good regulation and tracking performances. The robustness of the controller has also been studied when velocities of both internal and external fluid, and physical properties of the heat exchanger are subjected to sudden fluctuations. For noisy measurements and for practical implementation, the moving average filtering and Kalman estimation approaches that provide the required state temperatures to be used in the controller are discussed. The control by manipulating the jacket flow rate has also been considered to compare the respective benefits of both strategies.
\end{abstract}

Keywords : Distributed parameter system, boundary control, partial differential equation, heat exchanger, geometric control, Kalman filter

\section{Introduction}

In the industrial domain, the heat exchanger is an essential means of any policy for energy saving. The thermal energy used in the industrial processes deals with at least one heat exchanger. These systems are used in particular in the chemical, petrochemical and food industries. Concerning investment and quality, the advantages of the heat exchangers do not need any more to be proved. Nevertheless, these latter are frequently designed for open loop operation, so that the expected performances decrease quickly with time, hence the necessity to replace them frequently, which induces a negative impact at the economic level [Xia et al., 1991]. Consequently, their control becomes the alternate and adequate solution. However, it is well known that in addition to the unmodeled dynamics, heat exchangers are commonly connected to

*Author to whom the correspondence should be addressed : corriou@ensic.inpl-nancy.fr 
thermal equipments and this can cause sudden variations of the parameters such as inlet temperatures, physical properties of fluids, and flow rates of both fluids [Abdelghani-Idrissi et al., 2001a,b, Arbaoui et al., 2007, Xuan and Roetzel, 1993], so a robust controller is needed to achieve better performances.

In order to control the outlet temperature of heat exchanger, two approaches are possible for the controller synthesis. The first one uses a model considering the hypothesis of the homogeneity of temperature inside the tube [Abdelghani-Idrissi et al., 2001a, Alvarez-Ramirez et al., 1997, Davison et al., 1980, Dugdale and Wen, 2002, Katayama et al., December 1990, Malleswararao and Chidambaram, 1992]. This leads to work with a model given under the form of ordinary differential equations (ODE), i.e. a lumped parameter system (LPS), which allows us to apply the control theory developed for LPS [Corriou, 2004, Ray, 1989]. Christofides [2001] notes that for processes which are strongly spatially distributed, the discretization under the form of ODEs decreases the control performance.

The second approach, opposite to the first one, does not use the hypothesis of homogeneity of temperature inside the tube, and considers a model under the form of partial differential equations (PDE), i.e. a distributed parameter system (DPS), which suitably describes the spatial and dynamic behavior of the heat exchanger. Under some modeling hypotheses, the model of the dynamic behavior of a heat exchanger is often described by a quasi-linear partial differential equation [Christofides, 2001, Christofides and Daoutidis, 1996, Shang et al., 2005]. Thus, the use of a DPS model, when possible, allows the synthesis of a control system which improves the performances of the processes [Christofides, 2001].

Moreover some control strategies assume that the temperature of the jacket is homogeneous, i.e. not distributed, and the model used for the design of the control is reduced to a single partial differential equation which describes the distribution of the temperature inside the heat exchanger tube [Christofides, 2001, Christofides and Daoutidis, 1996, Lim and Fang, 1972, Ray and Ogunnaike, 1994, Shang et al., 2005]. This implies that the considered model does not correctly reflect the heat exchanger dynamics, so an accurate mathematical model, but not complicated, is desirable for control design purpose in order to achieve better performances.

Several PDE models of the heat exchanger have been studied in the literature, depending on the considered assumptions to deduce the differential equations and on the final use of the model. For control design, Lim and Fang [1972] developed and applied an optimal control theory for a heat exchanger given by a single PDE since the wall temperature is assumed spatially uniform. Pohjolainen and Lätti [1983] considered a linearized model of a counter current heat exchanger, obtained by linearizing a model given by a series of second-order PDEs that take the presence of the diffusion phenomena in the fluids into account, as an application to illustrate a robust control theory generalized to distributed parameter systems with boundary control and constant perturbations concerning the inlet conditions of the internal fluid. The stability of the same linearized model has been discussed by Xu and Gauthier [1991] and a state feedback control law is proposed. Another kind of heat exchanger that works with three input fluids, has been studied by Gauthier and Xu [1991]. The model given by a series of first-order PDEs, neglecting the diffusion phenomena, is linearized and controlled using $\mathcal{H}_{\infty}$ techniques. Hanczyc and Palazoglu [1995] have applied the sliding mode control theory to control a heat exchanger modeled by two nonlinear partial differential equations. Bittanti and Piroddi [1997] describe clearly their model but later use neural networks for their control strategy. Recently, Arbaoui et al. [2007] designed a predictive functional controller to control the outlet temperature of a counter-current tubular heat exchanger given by two PDEs (hot fluid, cold fluid) plus an ODE for the separating wall. However, the time evolution of the temperatures is approximated as a first order system. Alvarez et al. [2007] use transfer functions for their model of their heat exchanger, which is not a firm basis for the physical model of the process.

In fact, a model based on a single PDE describing the temperature evolution of the inner fluid does 
not correspond to a real heat exchanger. At the most, it corresponds to a tube exchanging heat with the environment for example by natural convection, and even in this case, the temperature of the wall should be considered as variable, so that the physical model would include one PDE for the inside of the tube and at least an ordinary differential equation for the wall. Thus a realistic model of a heat exchanger should include the two PDEs for the inner and external fluids.

This paper deals with the boundary geometric control of a counter-current heat exchanger, the control design of which is based on the use of a PDE model which considers the spatial distribution of the temperatures of the internal and external fluids. The objective is the control of the temperature of the fluid at the outlet of the exchanger by manipulating the temperature of the fluid in the jacket at the exchanger boundary. The temperature of the internal fluid at the exchanger inlet represents a disturbance influencing the process. Thus, a control problem has been formulated and the control law has been deduced by exploiting notions of geometric control such as exposed in [Christofides, 2001, Christofides and Daoutidis, 1996, Wu and Liou, 2001]. To evaluate the performances of the controller, simulation experiments concern set point tracking and disturbances taken as variations of the temperature of the internal fluid at the inlet of the exchanger. However, for practical implementation of the control law, two temperature measurements are required, so moving average filtering and Kalman estimation approaches are used and compared to estimate the necessary states in presence of measurement noise. A finitedimensional model with a lower order is considered for the design of the Kalman filter after discussing the process observability. The control by manipulating the jacket flow rate has also been considered to compare the respective benefits of both strategies depending on the choice of the manipulated variable.

The article is organized as follows : in section 2, the studied counter-current heat exchanger is presented and its dynamic model given as a system of two PDEs. Section 3 concerns the formulation of the control problem and the design of the boundary control laws. Section 4 is dedicated to simulation and to discussions concerning the numerical results in particular with respect to robustness and state estimation. Finally, a conclusion ends the article.

\section{Counter-current heat exchanger dynamic model}

\subsection{Description of the heat exchanger}

The process studied in this work corresponds to a tubular counter-current heat exchanger (Fig. 1). A liquid fluid of constant density $\rho$ and of heat capacity $C_{p}$ flows through the internal tube of a heat exchanger, of length $L$, with a constant velocity $v$. This fluid enters at temperature $T_{0}$ and exchanges heat with the second liquid or non condensating vapor fluid, of constant density $\rho_{e}$ and of heat capacity $C_{p_{e}}$, which flows counter-currently in the jacket with a velocity $v_{e}$. This fluid enters at temperature $T_{e_{L}}$. At the outlet of the exchanger, the internal fluid leaves at temperature $T_{L}$. In the present study, the internal and external cross sections $S$ and $S_{e}$ of the heat exchanger are supposed to be uniform and the surface area used for the heat transfer per unit length is $\mathcal{S}$. Both temperatures $T$ of the internal fluid and $T_{e}$ of the external fluid depend on time and spatial position along the tube.

\subsection{Model of the heat exchanger}

The energy balance of the heat exchanger, after classical simplifying hypotheses [Ray and Ogunnaike, 1994], gives the following partial differential equation for the internal tube

$$
\frac{\partial T(z, t)}{\partial t}=-v(t) \frac{\partial T(z, t)}{\partial z}+a\left[T_{e}(z, t)-T(z, t)\right], a=\frac{\tilde{h} \mathcal{S}}{\rho S C_{p}}
$$


and the following partial differential equation for the jacket

$$
\frac{\partial T_{e}(z, t)}{\partial t}=+v_{e}(t) \frac{\partial T_{e}(z, t)}{\partial z}+a_{e}\left[T(z, t)-T_{e}(z, t)\right], a_{e}=\frac{\tilde{h}_{e} \mathcal{S}}{\rho_{e} S_{e} C_{p_{e}}}
$$

$T$ and $T_{e}$ are the temperatures of the internal and external fluids, respectively, $a$ and $a_{e}$ are the heat transfer coefficients, $v$ and $v_{e}$ are the velocities, $\tilde{h}$ and $\tilde{h}_{e}$ are the overall heat transfer coefficients.

Each PDE requires an initial condition and a boundary condition to be fully defined. The studied heat exchanger is of counter-current type. For eq. (1) describing the temperature of the internal fluid, the boundary condition is usually specified at $z=0$ as the temperature of the liquid fluid entering the tube is in general known and measurable. Thus, at $z=0$, it gives

$$
T(0, t)=T_{0}(t)
$$

and most often the initial condition is some given temperature profile at $t=0$

$$
T(z, 0)=T^{*}(z)
$$

Similarly, for eq. (2), describing the distribution of temperature of the external fluid in the jacket, the boundary condition is the temperature of the entering fluid $T_{e_{L}}$, specified at $z=L$, consequently

$$
T_{e}(L, t)=T_{e_{L}}(t)
$$

while the initial condition (at $t=0$ ) is some given temperature profile

$$
T_{e}(z, 0)=T_{e}^{*}(z)
$$

Eqs. (1)-(6) constitute the dynamic model of the counter-current heat exchanger.

\section{Boundary control of the heat exchanger}

The aim of this work is the control of the internal fluid outlet temperature $T_{L}$ despite variations of the flow rate and of physical properties of the liquids. Thus, in this section, geometric control [Corriou, 2004, Isidori, 1995, Kravaris and Kantor, 1990a,b] is applied to control the outlet temperature of the internal liquid by manipulating either the temperature of the external fluid at the boundary or the jacket inlet flow rate. First, the partial differential equation for the internal tube is used to synthesize a state feedback controller that leads to a linear input-output system, which is the map between a new manipulated variable and an output variable. In the second step, in order to fully benefit from the power of the geometric control approach, a PI controller is designed for the input-output system to handle uncertainties (Fig. 2). The section starts with a review about the various approaches proposed for the control of heat exchanger in the literature before addressing our formulation of the boundary control problem based on the geometric control approach.

\subsection{Previous formulations of the control problem}

Several control studies of a heat exchanger [Christofides, 2001, Christofides and Daoutidis, 1996, Shang et al., 2005] have been performed considering only the PDE equation related to the internal fluid (eq. (1)) with the associated initial and boundary conditions, and the external fluid temperature is assumed homogenous. This dynamic model of the heat exchanger (1), (3),(4), that neglects the diffusion 
phenomenon, belongs to the class of systems given under the following state space form, which is quasilinear and affine with respect to the input $u(t)$

$$
\begin{aligned}
\frac{\partial x(z, t)}{\partial t} & =-v(t) \frac{\partial x(z, t)}{\partial z}+f(x)+g(x) u(t) \\
x(0, t) & =x_{0}(t) \\
x(z, t) & =x^{*}(z)
\end{aligned}
$$

where $x(z, t) \in \mathcal{H}\left[(0, t), \Re^{n}\right]$ is the state vector, $\mathcal{H}($.$) is the Hilbert space of the states defined in the interval$ $[0, L], z \in[0, L] \subset \Re, v$ is the velocity of the fluid, $x_{0} \in \Re^{n}$ is the vector of the boundary conditions, $x^{*} \in \Re^{n}$ is the vector of the initial conditions. $f$ and $g$ are sufficiently smooth vector functions.

The controlled output is chosen in a general way as a function of the states

$$
y(t)=h(x(z, t))
$$

where $h$ is a sufficiently smooth scalar function.

Two choices are possible for the manipulated variable. The first one consists in manipulating the variable $u(t) \in \Re^{m}$. Lim and Fang [1972] consider the same variable, which is assumed to be uniform, for an optimal control problem of the internal liquid outlet temperature. Christofides [2001], Christofides and Daoutidis [1996] propose to use the same value of the manipulated variable, in a distributed manner, to control an output obtained by an observation based on spatial averaging. The second possibility proposed and studied by Gundepudi and Friedly [1998] and Shang et al. [2005], consists in manipulating the fluid velocity $v(t)$ whereas the temperature of the jacket is uniform. Shang et al. [2005] use the same type of hypothesis for a plug-flow reactor. In the case of boundary control instead of a distributed control, $\mathrm{Wu}$ and Liou [2001] choose the outlet concentration of a plug-flow reactor as the controlled output

$$
y(t)=\left.h(x(z, t))\right|_{z=L}
$$

and compare two boundary control strategies for the application of the manipulated input, either only at the exit boundary of a reactor (at location $z=L$ ), or at both boundaries of a reactor with the same value of $u$ (at locations $z=0$ and $z=L$ ). This idea can be generalized to a flow system. Hanczyc and Palazoglu [1995] use the external fluid flow rate as a manipulated variable, while the outlet temperature of the external fluid is the controlled variable. Pohjolainen and Lätti [1983] consider a model that includes the diffusion phenomena to construct an adaptive robust controller to control the outlet temperature of the internal fluid by manipulating the inlet temperature and the flow rate of the external fluid. For the same model, the case where only the flow rate of the external fluid is manipulated to control the internal fluid outlet temperature has been addressed by Xu and Gauthier [1991]. A counter-current heat exchanger that works with three input fluids, one internal fluid and two external fluids, is studied by Gauthier and $\mathrm{Xu}$ [1991] where the internal fluid outlet temperature is the controlled variable and the two external fluids flows rates are the manipulated variables. Arbaoui et al. [2007], by considering a model given by three PDEs, use the external fluid flow rate as a manipulated variable to control the internal fluid outlet temperature.

In theory, the variable which is manipulated is the thermal power at the inlet of the outer tube of the heat exchanger, i.e. grossly the product of a flow rate and a difference of temperature. In practice, to control the outlet temperature of a counter-current heat exchanger, there are two possible strategies which are not equivalent. The first one is to use the inlet temperature of the external fluid, while the second is to manipulate its flow rate. Both possibilities are studied in the present paper with emphasis 
on control by means of the inlet temperature.

Let us first consider the case of flow rate as the manipulated input. If the flow rate becomes too small, the flow regime in the outer tube can be laminar instead of turbulent. A minimum bound is to set on this input. So a more realistic model should also include the influence of the flow rate on the parameters models, in particular the heat transfer coefficient [Xuan and Roetzel, 1993], and the tuning of the controller should vary with the flow rate, which is a difficult task [Abdelghani-Idrissi et al., 2001b, Arbaoui et al., 2007].

With the temperature as a manipulated input, it is possible to work at a constant large flow rate and the hydrodynamic regime is invariable. Physically, manipulating the temperature is nearly possible if this latter is the outlet of a process with fast dynamics like plate heat exchangers. Potential flow rate variations will be assumed as a disturbance that affects the system and needs to be rejected by the designed controller.

\subsection{Synthesis of the present control law}

\subsubsection{Control based on manipulated inlet temperature}

The dynamic model of the heat exchanger (1)-(6), belongs to the class of systems given under the state form (7). With regard to the techniques developed in section 3.1, the difference in the present approach lies in the fact that the influence of eq. (2) is taken into account with its initial and boundary conditions. The adopted strategy consists in controlling the temperature of the internal fluid at the outlet, i.e. $T(L, t)=T_{L}(t)$, by manipulating the inlet temperature of the external fluid $T_{e_{L}}(t)$, corresponding to the boundary condition (5). Due to eq. (2), it is noticeable that by manipulating the boundary condition of the jacket, given by eq. (5), a variation of the temperature of the external fluid $T_{e}$ along the jacket results. Thus, by denoting as $u$ the control variable and $y$ the controlled variable, the model of the heat exchanger (1)-(6), by adding eq. (9) of the output, takes the following form

$$
\begin{array}{ll}
\frac{\partial T(z, t)}{\partial t} & =-v(t) \frac{\partial T(z, t)}{\partial z}+a\left[T_{e}(z, t)-T(z, t)\right] \\
\frac{\partial T_{e}(z, t)}{\partial t} & =+v_{e}(t) \frac{\partial T_{e}(z, t)}{\partial z}+a_{e}\left[T(z, t)-T_{e}(z, t)\right] \\
T(0, t) & =T_{0}(t) \\
T_{e}(L, t) & =T_{e_{L}}(t)=u(t) \\
T(z, 0) & =T^{*}(z) \\
T_{e}(z, 0) & =T_{e}^{*}(z) \\
y(t) & =\left.T(z, t)\right|_{z=L}=T(L, t)
\end{array}
$$

For the synthesis of the geometric control law, the first stage is the determination of the relative order $r$ of the system. It is defined as the lowest order of derivation of the output $y(t)$ which depends explicitly linearly on the input $u(t)$ [Christofides, 2001, Christofides and Daoutidis, 1996, Corriou, 2004, Isidori, 1995]. Being given the relative order of the heat exchanger model, the successive time derivatives of the output $y(t)$ are considered. Thus for system (10), the first derivative of the outlet temperature of the internal fluid is

$$
\frac{d y(t)}{d t}=\left.\frac{d T(z, t)}{d t}\right|_{z=L}=-\left.v(t) \frac{\partial T(z, t)}{\partial z}\right|_{z=L}+a[\underbrace{T_{e}(L, t)}_{u(t)}-T(L, t)]
$$


The manipulated input $u(t)$ appears linearly in the first time derivative of the output, as for the heat exchanger $a \neq 0$, so that the relative order of the present system is $r=1$. It means that a first order linear behavior can be imposed for the pair [desired output $\nu(t)$-output $y(t)$ ] in closed loop, being given the following control law (12) deduced from eq. (11) such that

$$
u(t)=\frac{1}{a}\left[\frac{1}{\tau}(\nu(t)-T(L, t))+\left.v(t) \frac{\partial T(z, t)}{\partial z}\right|_{z=L}+a T(L, t)\right]
$$

where $\tau$ is the desired time constant for the closed loop linear behavior between the input $\nu(t)$ and output $y . \nu(t)$ is called an external input for the system. It can be noticed that the first derivative of the exiting fluid at location $z=L$ intervenes in the control law. The control robustness dealing with problems of model and parameter uncertainty and unmodeled dynamics, is provided in (12) through application of the linear control theory to the resulting linear input-output system, by adopting the control strategy given by Fig. 2. A Lyapunov-based control approach is proposed by Christofides and Daoutidis [1998] for

this purpose. In our case, the robustness is ensured by the external input $\nu(t)$ as a PI controller [Kravaris and Kantor, 1990b] as follows

$$
\nu(t)=K_{c}\left[\left(y^{d}(t)-y(t)\right)+\frac{1}{\tau_{I}} \int_{0}^{t}\left(y^{d}(\xi)-y(\xi)\right) d \xi\right]
$$

where $K_{c}, \tau_{I}$ and $y^{d}(t)$ are respectively the proportional gain, integral time constant and desired output. The transfer function of the closed loop system is then the following

$$
\frac{Y(s)}{Y^{d}(s)}=\frac{\frac{K_{c}}{\tau} s+\frac{K_{c}}{\tau \tau_{I}}}{s^{2}+\left(\frac{1}{\tau}+\frac{K_{c}}{\tau}\right) s+\frac{K_{c}}{\tau \tau_{I}}}
$$

The scalar parameters $K_{c}, \tau_{I}$ and $\tau$ are chosen in order for the denominator to approach a polynomial minimizing an ITAE criterion [Corriou, 2004]; we verify that the following polynomial is Hurwitz (the poles have a negative real part) to ensure the closed loop stability related to the roots of the characteristic equation

$$
s^{2}+\left(\frac{1}{\tau}+\frac{K_{c}}{\tau}\right) s+\frac{K_{c}}{\tau \tau_{I}}=0
$$

\subsubsection{Control based on manipulated flow rate}

When the external fluid flow rate is chosen as the manipulated variable, the inlet temperature $T_{e}(L, t)$ for the outer tube is constant except in case of temperature disturbances. Thus Equation (2) considered at $z=L$ is no more a PDE, but algebraic, and can be rewritten as

$$
0=\left.v_{e}(t) \frac{\partial T_{e}(z, t)}{\partial z}\right|_{z=L}+a_{e}\left[T(L, t)-T_{e}(L, t)\right]
$$

Note that $T(L, t)$ is the desired temperature at the outlet of the inner tube so that the input directly influences the output without any apparent dynamics, the relative order is equal to $r=0$. It results that the velocity $v_{e}(t)$, equivalent to the flow rate, can be expressed as

$$
v_{e}(t)=\frac{a_{e}\left[y^{d}(t)-T_{e}(L, t)\right]}{\left.\frac{\partial T_{e}(z, t)}{\partial z}\right|_{z=L}}
$$


This control law is valid for a perfect model and in absence of disturbances. The partial derivative of the denominator depends implicitly on the desired output $y^{d}(t)=T(L, t)$ and can be calculated at steady state for different values of $y_{d}$. The dependence of $v_{e}(t)$ on the desired temperature $T(L, t)$ is given in Fig. 3 for the heat exchanger parameters given in section 4 . However, if this value of $v_{e}(t)$ is strictly applied as such, there is no dynamic consideration and no rejection of any disturbance. It would simply be an open-loop control. A dynamic correction term accounting for the disturbances and error modeling could be added to the previously calculated value by means of a PID controller for example.

Another approach for manipulating the external fluid flow rate is developed in the following. Since the set of PDEs of the considered heat exchangers is hyperbolic, discontinuities in the state profiles may occur [Gundepudi and Friedly, 1998]. Thus, to have $v_{e}$ well-defined, the controlled output is evaluated at $z=L-\epsilon$ and not at $z=L$ [Gundepudi and Friedly, 1998], where $\epsilon$ is a small length increment. This amounts to avoid considering the algebraic equation (16) and to retain the character of the original PDE (2). Again a geometric control law is developed and the relative order of the output is equal to $r=2$. Thus, the calculation of the successive time derivatives of the controlled output $y(t)=T(L-\epsilon, t)$ leads to the following control law

$$
\begin{aligned}
u(t)= & \frac{1}{\left.a \beta_{2} \frac{\partial T_{e}}{\partial z}\right|_{z=L-\epsilon}} \times\left\{\nu(t)-\left.\beta_{2} v^{2} \frac{\partial^{2} T}{\partial z^{2}}\right|_{z=L-\epsilon}+\left.\beta_{2} a v \frac{\partial T_{e}}{\partial z}\right|_{z=L-\epsilon}-\left.\left(2 \beta_{2} a v-\beta_{1} v\right) \frac{\partial T}{\partial z}\right|_{z=L-\epsilon}\right. \\
& \left.+\left(\beta_{2} a^{2}+\beta_{2} a a_{e}-\beta_{1} a\right)\left(T_{e}(L-\epsilon, t)-T(L-\epsilon, t)\right)-T(L-\epsilon, t)\right\}
\end{aligned}
$$

where $\beta_{1}$ and $\beta_{2}$ are adjustable controller parameters. In the same way, for robustness purposes, the external input $\nu(t)$ can be defined by a PI controller, and the closed loop system transfer function will be

$$
\frac{Y(s)}{Y^{d}(s)}=\frac{K_{c}\left(\tau_{I} s+1\right)}{\tau_{I} \beta_{2} s^{3}+\tau_{I} \beta_{1} s^{2}+\left(\tau_{I}+K_{c} \tau_{I}\right) s+K_{c}}
$$

\section{Simulation results}

In order to evaluate the performance of the control system, several simulation runs are performed. Thus, the model of the exchanger (10) has been discretized according to the following backward finite difference scheme

$$
\frac{\partial x(z, t)}{\partial z} \approx \frac{x\left(z_{i}, t\right)-x\left(z_{i-1}, t\right)}{\Delta z}, i=1, \ldots, N
$$

where $N$ is the number of discretization points, $\Delta z=L / N$ is the distance between two adjacent discretization points, and $z_{i}=i \Delta z(i=0, \ldots, N)$. Notice that $z_{0}=0$ and $z_{N}=L$.

Similarly, the discretization of the control law (12), according to eq. (20), makes use of the term

$$
\left.\frac{\partial T(z, t)}{\partial z}\right|_{z=L} \approx \frac{T(L, t)-T(L-\Delta L, t)}{\Delta L}
$$

Consequently, at a practical level, the implementation of the control $u(t)$ requires the use of two sensors placed at two adjacent positions defined by $z=L-\Delta L(\Delta L \neq \Delta z)$ and $z=L$, or the estimation of the states at these locations.

In the case of flow control, in practice, $\epsilon$ can be taken as zero without any trouble in control law (18). Furthermore, five measurements or estimations are required to evaluate the derivative terms of the 
control law, i.e. four measured inner temperatures $T(L, t), T(L-\Delta L, t), T(L-2 \Delta L, t), T(L-3 \Delta L, t)$, and one measured outer temperature $T_{e}(L-\Delta L, t)$ as $T_{e}(L, t)$ is known and imposed. $\Delta L$ is a length increment sufficiently large to suppress the influence of measurement noise (see section 4.1.4 for a sounder discussion).

The employed method of finite differences leads to a finite-dimensional approximation of the form

$$
\begin{aligned}
& \dot{X}(t)=A X(t)+B u(t)+E T_{0}(t) \\
& y(t)=C X(t)
\end{aligned}
$$

where

$$
X(t)=\left[\begin{array}{llllll}
T_{1}(t) & \ldots & T_{N}(t) & T_{e_{1}}(t) & \ldots & T_{e_{N}}(t)
\end{array}\right]^{T}
$$

with $T_{i}(t) \equiv T\left(z_{i}, t\right)$ and $T_{e_{i}}(t) \equiv T_{e}\left(z_{i}, t\right)(i=1, \ldots, N)$, while the matrices $A \in \Re^{N \times N}, B \in \Re^{N \times 1}, E \in$ $\Re^{N \times 1}$ and $C \in \Re^{1 \times N}$ are derived from the discretization process. Note that the number of discretization points $N$ must be sufficiently large to ensure a good approximation of the original system.

In the simulation conditions, the nominal parameters of the studied heat exchanger are $a=2.92 \mathrm{~s}^{-1}$, $v=1 \mathrm{~m} \cdot \mathrm{s}^{-1}, a_{e}=5 \mathrm{~s}^{-1}, v_{e}=2 \mathrm{~m} \cdot \mathrm{s}^{-1}$ and $L=1 \mathrm{~m}$. For steady-state study, it is considered that the internal fluid enters the exchanger at constant temperature $T_{0}=T(0, t)$ equal to $25^{\circ} \mathrm{C}$ (boundary condition at $z=0)$ and the external fluid enters at constant temperature $T_{e_{L}}=T_{e}(L, t)$ equal to $50^{\circ} \mathrm{C}$. The initial conditions are taken as the spatial profiles of both fluid temperatures obtained at steady-state by making the time derivatives of model (10) equal to zero. These spatial profiles are given by Fig. 4 .

\subsection{Control based on manipulated inlet temperature}

Following the tuning procedure described at the end of subsection 3.2.1, the control law parameters obtained are : $\tau=1 \mathrm{~s}, K_{c}=7.8$ and $\tau_{I}=1.03 \mathrm{~s}$. Note that, for all simulation, the control is held constant over the sampling period equal to $0.02 \mathrm{~s}$. All tests are performed by assuming a number of discretization points $N=100$.

\subsubsection{Tracking problem}

In the first simulation run, the reference input tracking capabilities of the controller are studied. Thus, two set point steps have been specified at times $t=1 \mathrm{~s}$ and $t=5 \mathrm{~s}$ corresponding respectively to $y^{d}(t)=50^{\circ} \mathrm{C}$ and $y^{d}(t)=30^{\circ} \mathrm{C}$. To avoid the consequences due to brutal set point steps, the set point has been filtered by a first order filter with a time constant equal to $0.2 \mathrm{~s}$. On Fig. 5 , it is clear that the output $y(t)$ follows perfectly the imposed set point whereas the control moves of $u(t)$ are physically acceptable.

The examination of Fig. 5 shows that a crossover effect, i.e. the outer temperature being colder than the inner temperature, appears after time $t=5 \mathrm{~s}$ of the negative set point variation of $-20^{\circ} \mathrm{C}$. The demonstration of the crossover is given in Fig. 6 where normal temperature profiles are present just before $t=5 \mathrm{~s}$, then the crossover propagates itself during some time, and disappears after about $1.5 \mathrm{~s}$. The crossover is always observed when negative steps of large amplitude on the inner temperature set point are imposed, but not for positive steps. When negative steps are desired for the temperature set point, very stiff negative variations of the manipulated temperature at the inlet of the outer tube can result in a transient crossover which in general is all the more pronounced as the considered spatial location is close to the inlet of the outer tube. 


\subsubsection{Disturbance rejection}

The second performed test concerns the problem of disturbance rejection. The performances of the controller are thus evaluated with respect to changes of the fluid temperature at the inlet of the heat exchanger which is a disturbance for the system. For that reason, a step of $-20 \%$ of the temperature of the entering liquid fluid (at $z=0$ ) is imposed as a disturbance at time $t=5 \mathrm{~s}$, after having imposed a step set point at time $t=1 \mathrm{~s}$ corresponding to $y^{d}(t)=50^{\circ} \mathrm{C}$. It appears that the controlled output (Fig. 7) is not influenced by the disturbance, the effect of which is compensated by the variation of the manipulated input (Fig. 7). On the other hand, if the temperatures at positions $z=0,0.25,0.50,0.75,1 \mathrm{~m}$ (Fig. 8) are examined, it can be noticed that the step disturbance visible at $z=0$ is attenuated as the position moves from the inlet $(z=0)$ towards the outlet $(z=L=1)$ where it becomes nearly invisible at the outlet of the heat exchanger. Again, the profiles of temperature after successively the step set point and the step disturbance (Fig. 9) are typical of the behavior of a counter-current heat exchanger.

\subsubsection{Robustness}

The robustness of the controller is investigated, thus a perturbed model is simulated for different changes of the system parameters, i.e. heat transfer coefficients $a, a_{e}$ and velocities $v, v_{e}$, assumed as parametric uncertainties or as sudden fluctuations since heat exchangers are commonly connected to other thermal equipments and this can affect the physical parameters and the operating flow rates. For this test, any parameter change occurs at $t=5 \mathrm{~s}$ after having imposed a step set point $y^{d}(t)=50^{\circ} \mathrm{C}$ at time $t=1 \mathrm{~s}$. Figs. 10-12 show the performance of the control system for different variations of the heat exchanger parameters. It should be noted that in spite of these large parametric uncertainties, the controlled output still tracks very correctly its reference set point with admissible variations of the manipulated variable.

\subsubsection{Measurement noise and sensor location}

In actual practice, the measurements are often corrupted by significant measurement noise, which is a stochastic and nondeterministic error of the sensors that cannot be predicted. Thus in the case where noises are not considered at the design stage, the control strategy will lack robustness. So, before discussing the techniques used herein to provide acceptable estimates of the required state temperatures, it is judicious to investigate the influence of the location of the sensor that measures the temperature at position $z=L-\Delta L$ since the sensitivity to the measurement noise of the derivative term in the control law (12), given by eq. (21), will be influenced by this location. For this purpose, different values of $\Delta L$ are considered and taken equal to $k \Delta z$ with $k$ is an integer, and the available measurements are corrupted with Gaussian white noise signals of standard deviation equal to $0.2^{\circ} \mathrm{C}$. Thus a set point step corresponding to $y^{d}(t)=50^{\circ} \mathrm{C}$ and a step of $-20 \%$ of the temperature of the entering fluid (at $z=0$ ) are specified at $t=1 \mathrm{~s}$ and $t=5 \mathrm{~s}$, respectively. It is noted from Fig. 13 that by moving the sensor from the outlet of the heat exchanger, i.e. by increasing $k$, the effect of the measurement noise decreases and the control performance is improved. Furthermore, the increase of $\Delta L$ does not have any negative effect on the control problem since in a heat exchanger, in general, the spatial profiles of temperatures are smooth, so increasing $k$ in a small range up to about 10 does not deteriorate significantly the derivative approximation. Consequently, from a practical point of view, for the implementation of the control system, the two sensors must be sufficiently distant. However, it is worth noting that the implementation depends on the available measurements, thus two situations are possible. When both measurements at $z=L-\Delta L$ and $z=L$ are available, then either a filtering or an estimation technique can be adopted, but if only 
the measurement at $z=L$ (output) is available, an estimation technique is necessary to estimate the measurement at $z=L-\Delta L$. These situations are examined below by considering that the second sensor is located at a position defined by $\Delta L=5 \Delta z$.

Consider also an error on the position of the second sensor previously assumed to be located at $z=L-\Delta L$. Assume that the actual sensor position is given by $\Delta L^{\prime} \neq \Delta L$. Two different values of $\Delta L^{\prime}$ are considered corresponding to an error of $\pm 20 \%$, and a set point step corresponding to $y^{d}(t)=50^{\circ} \mathrm{C}$ is specified at $t=5 \mathrm{~s}$. In these conditions, even in presence of noise, the set point is correctly tracked (Fig. 14), demonstrating a satisfactory robustness with respect to the error of location of the sensor.

Filtering Both measurements are assumed to be available at positions $z=L-\Delta L$ and $z=L$. In this case, for an on-line implementation of the control system, a simple filtering or smoothing approach can be used to restore the actual measurements. Thus, it is assumed that the two corrupted measurements are filtered by a moving average filter with a forgetting factor. The obtained results (Figs. 15-17) show that the controller behaves suitably and achieves satisfactory performance since the controlled output presents acceptable deviations with respect to the imposed set point (Fig. 16) with moderate solicitations of the manipulated variable (Fig. 17).

State estimation State estimation is widely used for online-implementation of state-space control laws since these methods demand the knowledge of many of the state variables, if not all of them. In this way, several techniques have been introduced to estimate variables from the available measurements. With respect to the estimation techniques performed up to now, the Kalman filter is one of the most widely diffused estimators [Simon, 2006]. This latter will be considered, in this section, to perform an estimation of the required state temperatures to be used in the controller law (the temperature at $z=L-\Delta L$ and $z=L$ ) by using the available measurements. Thereafter, it is assumed that only the output temperature $y$ (temperature at $z=L$ ) is available. So the estimation of the unmeasured variable (the temperature at $z=L-\Delta L)$ is performed using the two available measurements : temperature at $z=L$ and the measured disturbance $T_{0}$.

Before designing the Kalman filter, it is necessary to study the observability of the system. The system being of distributed nature, observability is not only affected by the choice of sensors but by the sensor location [Ray, 1989, Waldraff et al., 1998]. As, in general, it is quite difficult to prove observability for a distributed parameter system, and in most cases a discretization scheme is applied in order to get a finite-dimensional approximation, then different observability measures can be applied [Waldraff et al., 1998]. However, a drawback is associated with this approach since the observability, which depends only on the location of sensors, may depend on the discretization method, the number and location of discretization points, so neglecting the infinite dimensional nature of the original system may lead to erroneous conclusions concerning the fundamental control theoretical properties [Christofides, 2001]. Concerning the observability study of the heat exchanger, as the dynamic model (10) is a set of two first-order hyperbolic equations, the checkout of the general conditions for observability of first-order PDE systems discussed by Ray [1989, page 293] confirms that the process is observable. Based on the approximate model (22), and in presence of process and measurements noises, the process takes the form

$$
\begin{aligned}
& \dot{X}(t)=A X(t)+B u(t)+E T_{0}(t)+\zeta(t) \\
& y(t)=C X(t)+\eta(t)
\end{aligned}
$$

where $\zeta$ is a zero mean Gaussian process noise with covariance $Q$, and $\eta$ is a zero mean Gaussian measurement noise with covariance $R$. 
For implementation purpose, a lower order than that of model (24) will be considered for Kalman filter design, resulting in the following finite-dimensional approximate model

$$
\begin{aligned}
\dot{\hat{X}}(t) & =\hat{A} X(t)+\hat{B} u(t)+\hat{E} T_{0}(t) \\
y(t) & =\hat{C} X(t)
\end{aligned}
$$

where $\hat{A} \in \Re^{N_{f} \times N_{f}}, \hat{B} \in \Re^{N_{f} \times 1}, \hat{E} \in \Re^{N_{f} \times 1}$ and $\hat{C} \in \Re^{1 \times N_{f}}$ are the matrices of the reduced model derived by considering $N_{f}$ discretization points with $N_{f}<N$.

As the process is in continuous form and measurements are in discrete form, the Kalman filter is used in its discrete-continuous formulation [Corriou, 2004, Simon, 2006]. The state estimator is based on three measured variables $u, T_{0}$ and $y$ and yields the estimated state vector $\hat{X}$ where the matrix gain of the estimator is evaluated using the Kalman filtering algorithm. The equations for the Kalman filter are given by a set of prediction and correction equations as shown in the following :

Prediction phase : the following state and covariance equations

$$
\begin{aligned}
\dot{\hat{X}}(t) & =\hat{A} \hat{X}(t)+\hat{B} u(t)+\hat{E} T_{0}(t) \\
P(t) & =\hat{A} P(t)+P(t) \hat{A}^{T}+Q
\end{aligned}
$$

are integrated from time $k-1$ to $k$ in order to obtain the estimate $\hat{X}_{k / k-1}$ and $P_{k / k-1} \cdot \hat{X}_{k / k-1}$ denotes the estimate of state $X$ at $t=k$ from given information at $t=k-1$, and $P$ is the covariance matrix.

Correction phase : Correct the prior estimates of states at $k$ and update the covariance matrix

$$
\begin{aligned}
K_{k} & =P_{k / k-1} C^{T}\left[C P_{k / k-1} C^{T}+R\right]^{-1} \\
\hat{X}_{k / k} & =\hat{X}_{k / k-1}+K_{k}\left[y_{k}-C \hat{X}_{k / k-1}\right] \\
\hat{P}_{k / k} & =\left[I-K_{k} C\right] \hat{P}_{k / k-1}
\end{aligned}
$$

where the initial value of $P$ as well as the value of $R$ and $Q$ are the Kalman filter parameters.

The Kalman filter will provide the estimated temperatures at locations $z_{j}^{\prime}=j \Delta L=j k \Delta z(j=$ $\left.1, \ldots, N_{f}\right)$ with $\Delta L=L / N_{f}$. Recall that the required estimates measurements for control law implementation are the state temperatures corresponding to location $z=L-\Delta L$, with for example $\Delta L=5 \Delta z$, and $z=L$.

For simulation purpose, the appropriate values of the Kalman filter parameters $R, Q$ and $P(0 / 0)$ are set using trial and error approach and the final retained parameters were set as follows :

$$
R=0.01, Q=0.05 I_{\left(2 N_{f} \times 2 N_{f}\right)}, P(0 / 0)=0.01 I_{\left(2 N_{f} \times 2 N_{f}\right)}
$$

where $I$ is an identity matrix. The initial conditions $\hat{X}(0)$ are the steady state profiles. Note that for the filtering equations, a finite-dimensional approximate model with $N_{f}=40$ is assumed. By decreasing $N_{f}$ below that threshold, the estimation error increases, and the performance is reduced. For the real process, $N=100$ points of discretization are considered as in preceding sections.

In addition, taking into account eq. (25), in order to minimize the effect of the measurements errors related to the temperature $T_{0}$ on the state estimation process, it is judicious to perform a simple filtering before proceeding to the Kalman filtering. In this work, this is done by assuming a moving average filter with a forgetting factor.

The obtained simulation results are depicted in Figs. 18-20. In Fig. 18, at $z=L-\Delta L$ the designed Kalman filter allows an acceptable estimation of the unmeasured variable that leads to an acceptable 
tracking performance (Fig. 19) and admissible variations of manipulated variable (Fig. 20).

In conclusion, from the obtained results, the Kalman filter allows to achieve better performances than those obtained by using the moving average filter. Thus, the comparison between Fig. 16 and Fig. 19, and between Fig. 17 and Fig. 20 shows that the output tracks very correctly the imposed set point, and the manipulated variable is more damped in the case of use of the Kalman filter.

Finally, as mentioned above, the heat exchanger is subject to sudden parameters changes, thus the controller performance is studied with Kalman estimator in presence of model-process mismatch related to the parameters changes. Thus, Figs. 21-23 give the obtained results for a set point step, corresponding to $y^{d}(t)=50^{\circ} \mathrm{C}$, performed at $t=1 \mathrm{~s}$ followed by a parameter change at $t=5 \mathrm{~s}$. In spite of the parameter change, the Kalman estimator ensures a good estimation of the temperature at position $z=L-\Delta L$ (Fig. 21), which leads to satisfactory tracking (Fig. 22) with admissible control variations (Fig. 23).

\subsection{Control based on manipulated outer flow rate}

In this section, the performance of the control system based on the manipulation of the external flow rate, i.e. control law (18), is evaluated. The controller parameters obtained by the previously described tuning procedure are : $\tau_{1}=3.06 \mathrm{~s}, \tau_{2}=0.5 \mathrm{~s}^{2}, K_{c}=18.91$, and $\tau_{I}=0.882 \mathrm{~s}$. As indicated above, the ability of the controller to track a given reference input depends on the inlet temperature of the external fluid $T_{e}(L, t)$ that is considered as constant. In this simulation run, it is taken equal to $50^{\circ} \mathrm{C}$, and the same simulation conditions as in the preceding sections are considered. A set point step corresponding to $y^{d}(t)=47^{\circ} \mathrm{C}$ is specified at $t=1 \mathrm{~s}$, followed by a step of $-20 \%$ at $t=15 \mathrm{~s}$ of the inlet temperature of the internal fluid $T(0, t)$ that represents the disturbance affecting the system. Fig. 24 shows that the controller ensures both tracking and regulation, but the tracking is performed more slowly than in the case of the manipulated inlet temperature, and a significant effect of the disturbance is noted on the controlled output. In addition, a stiff variation of the manipulated variable is required for rejecting the perturbation effect. Adjusting the controller parameters in order to decrease the settling time tends to provoke oscillations of the output, especially for disturbance rejection.

\section{Conclusion}

In the present article, a boundary control designed in the framework of differential geometry has been applied to control the temperature of the liquid fluid at the outlet of a heat exchanger. To ensure the robustness in front of modeling errors, an external input is defined by means of a PI controller. The control is based on a PDE model, with two partial differential equations describing the spatial variations of the internal and external temperatures, characterizing the dynamic behavior of the heat exchanger. The simulation results clearly show that the outlet temperature of the internal fluid can be perfectly controlled by manipulating the temperature of the external fluid at the inlet, thus corresponding to a boundary control. The regulation and the tracking are perfectly performed. The noticeable advantage of the boundary control of the temperature of the internal fluid, at the outlet of the heat exchanger, by manipulating the boundary condition in the jacket, lies in the insensitivity of the controlled output with respect to the disturbances influencing the process. In addition, the proposed strategy has proved to be robust, even when confronted to significant modeling errors, i.e. parameter variations of both fluids and unmodeled dynamics, and the control system can be applied by use of two distant sensors. The problem of the presence of the measurements errors, due the noise effect and to the sensor location, is examined. According to the available measurements, two approaches are examined. Thus the moving average filtering approach is considered when the two required state temperatures are available, while the 
Kalman filter is used when only the outlet temperature is available. These approaches are discussed and then compared. The obtained results show that, even if both approaches lead to acceptable performance, the control system based on the Kalman filter allows us to achieve better performance.

A control strategy based on the flow rate of the external fluid as a manipulated variable is also designed and is compared to that deduced from the use of the inlet temperature of the external fluid. Simulation results show that this latter variable gives better performance than the flow rate.

A double pipe heat exchanger and a tubular reactor with a jacket are relatively similar from the point of view of their models. In the energy balance of a tubular reactor for the internal tube, the chemical reaction must be considered. Furthermore, mass balances are considered for the chemical species which of course do not exist for a heat exchanger. The technique which is employed in the present article could be exactly used in the case of the tubular reactor by neglecting the term of heat of reaction in the control law and thus considering that the chemical reaction is a supplementary disturbance for the process that is to be rejected. Another possibility resides in the development of an extended control law where the term of heat of reaction is considered, i.e. the model of the tubular reactor is used to develop the control law. This presents no real difficulty, as the manipulated temperature at the inlet of the external tube intervenes in the same way in the models of the heat exchanger and of the tubular reactor. However, for this second control law, an observer would be necessary to estimate the concentrations which are part of the reaction heat term, otherwise the concentrations at the outlet would have to be measured.

\section{Références}

M. A. Abdelghani-Idrissi, M. A. Arbaoui, L. Estel, and J. Richalet. Predictive functional control of a counter current heat exchanger using convexity property. Chemical Engineering and Processing, 40 : 449-457, 2001a.

M. A. Abdelghani-Idrissi, F. Bagui, and L. Estel. Analytical and experimental response time to flow rate step along a counter flow double pipe heat exchanger. International Journal of Heat and Mass Transfer, 44 :3721-3730, 2001b.

J.D. Alvarez, L.J. Yebra, and M. Brenguel. Repetitive control for tubular heat exchangers. Journal of Process Control, 17 :689-701, 2007.

J. Alvarez-Ramirez, I. Cervantes, and R. Femat. Robust controllers for a heat exchanger. Industrial $\mathcal{E}^{\circ}$ Engineering Chemistry Research, 36 :382-388, 1997.

M. A. Arbaoui, L. Verniéres-Hassimi, D. Seguin, and M. A. Abdelghani-Idrissi. Counter-current tubular heat exchanger : modeling and adaptive predictive functional control. Applied thermal Engineering, $27: 2332-2338,2007$.

D. Bittanti and L. Piroddi. Nonlinear identification and control of a heat exchanger : A neural network approach. Journal of The Franklin Institute, 334B :135-153, 1997.

P. D. Christofides. Nonlinear and Robust Control of PDE Systems : Methods and Applications to Transport-Reaction Processes. Birkhäuser, Boston, 2001.

P. D. Christofides and P. Daoutidis. Feedback control of hyperbolic PDE systems. AIChE Journal, 42 : 3063-3086, 1996.

P. D. Christofides and P. Daoutidis. Robust Control of hyperbolic PDE Systems, volume 53. Chemical Engineering Science, 1998. 
J. P. Corriou. Process control - Theory and applications. Springer, London, 2004.

E. J. Davison, P. A. Taylor, and J. D. Wright. On the application of tuning regulators to control a commercial heat exchanger. IEEE Transactions on Automatic Control, 25 :361-375, 1980.

D. Dugdale and P. Wen. Controller optimization of a tube heat exchanger. In Proceedings of the 4 th World Congress on Intelligent Control and Automation, pages 54-58, Shanghai, P. R. China, June 10-14, 2002.

J. P. Gauthier and C. Z. Xu. $\mathcal{H}_{\infty}$ control of a distributed parameter system with non-minimum phase. International Journal of Control, 53 :45-79, 1991.

P. K. Gundepudi and J. C. Friedly. Velocity control of hyperbolic partial differential equation systems with single characteristic variable. Chemical Engineering Science, 53 :4055-4072, 1998.

E. M. Hanczyc and A. Palazoglu. Nonlinear control of a distributed parameter process : the case of multiple caracteristics. Industrial \& Engineering Chemistry Research, 34 :4406-4412, 1995.

A. Isidori. Nonlinear control systems. Springer-Verlag, New York, 1995.

T. Katayama, T. Itoh, M. Ogawa, and H. Yamamoto. Optimal tracking control of a heat exchanger with change in load condition. In Proceeding of the 29th Conference on Decision and Control, pages 1584-1589, Honolulu, Hawaii, December 1990.

C. Kravaris and J. C. Kantor. Geometric methods for nonlinear process control. 1. background. Industrial \& Engineering Chemistry Research, 29 :2295-2310, 1990a.

C. Kravaris and J. C. Kantor. Geometric methods for nonlinear process control. 2. controller synthesis. Industrial 8 Engineering Chemistry Research, 29 :2310-2323, 1990 b.

H. C. Lim and R. J. Fang. Optimal feedback control of a class of linear tubular processes. AIChE Journal, pages 282-286, 1972 .

Y. S. N. Malleswararao and M. Chidambaram. Non-linear controllers of a heat exchanger. Journal of Process Control, 2 :17-21, 1992.

S. Pohjolainen and I. Lätti. Robust controller for boundary control systems. International Journal of Control, 38 :1189-1197, 1983.

W. H. Ray. Advanced Process Control. Butterworths, Boston, 1989.

W.H. Ray and B.A. Ogunnaike. Process Dynamics, Modeling and Control. Oxford University Press, 1994.

H. Shang, J.F. Forbes, and M. Guay. Feedback control of hyperbolic distributed parameter systems. Chemical Engineering Science, 60 :969 - 980, 2005.

D. Simon. Optimal State Estimation : Kalman, $H_{\infty}$, and Nonlinear Approaches. John Wiley \& Sons, New Jersey, 2006.

W. Waldraff, D. Dochain, S. Bourrel, and A. Magnus. On the use of observability measures for sensor location in tubular reactor. Journal of Process Control, 8 :497-505, 1998.

W. Wu and C. Liou. Output regulation of nonisothermal plug-flow reactors with inlet perturbations. Computers and Chemical Engineering, 25 :433-443, 2001. 
L. Xia, J.A. De Abreu-Garcia, and T. T. Hartley. Modelling and simulation of a heat exchanger. In IEEE International Conference on Systems Engineering, pages 453-456. 1-3 August, 1991.

C. Z. Xu and J. P. Gauthier. Analyse et commande d'un échangeur thermique à contre-courant. APII, $25: 377-396,1991$.

Y. Xuan and W. Roetzel. Dynamics of shell-and-tube heat exchangers to arbitrary temperature and step flow variations. AIChE Journal, 39 :413-421, 1993.

\section{Nomenclature}

A state matrix of the finite-dimensional approximate system

$\hat{A} \quad$ state matrix of the finite-dimensional approximate system with a lower model

a heat transfer coefficient of the internal liquid $\left[\mathrm{s}^{-1}\right]$

$a_{e} \quad$ heat transfer coefficient of the external liquid $\left[\mathrm{s}^{-1}\right]$

$B \quad$ input matrix of the finite-dimensional approximate system

$\hat{B} \quad$ input matrix of the finite-dimensional approximate system with a lower model

$C_{p} \quad$ heat capacity of the internal liquid $\left[{\left.\mathrm{J} . \mathrm{kg}^{-1} \cdot \mathrm{K}\right]}\right.$

$C_{p_{e}} \quad$ heat capacity of the the external liquid $\left[\mathrm{J} \cdot \mathrm{kg}^{-1} \cdot \mathrm{K}\right]$

$E \quad$ disturbance matrix of the finite-dimensional approximate system

$\hat{E} \quad$ disturbance matrix of the finite-dimensional approximate system with a lower model

$f, g \quad$ smooth vector functions

$\mathcal{H} \quad$ Hilbert space

$h \quad$ smooth scalar function

$\tilde{h} \quad$ overall heat transfer coefficient of the internal fluid $\left[\mathrm{W} \cdot \mathrm{m}^{-2} \cdot \mathrm{K}^{-1}\right]$

$\tilde{h}_{e} \quad$ overall heat transfer coefficient of the external fluid $\left[\mathrm{W} \cdot \mathrm{m}^{-2} \cdot \mathrm{K}^{-1}\right]$

I identity matrix

$k \quad$ integer number

$K \quad$ Kalman filter gains matrix

$K_{c} \quad$ proportional gain of the PI controller

$L \quad$ length of the heat exchanger [m]

$m \quad$ number of manipulated variables

$N \quad$ number of discretization points

$n \quad$ number of state variables

$P \quad$ covariance matrix

$\hat{P} \quad$ estimate of the covariance matrix

$Q \quad$ covariance matrix of process noise

$R \quad$ covariance matrix

$\Re \quad$ set of real numbers

$r \quad$ relative order

$S \quad$ internal cross section $\left[\mathrm{m}^{2}\right]$

$S_{e} \quad$ external cross section $\left[\mathrm{m}^{2}\right]$

$\mathcal{S} \quad$ heat transfer surface area per unit length [m]

$s \quad$ Laplace operator

$T \quad$ internal fluid temperature $\left[{ }^{\circ} \mathrm{C}\right]$

$T_{0} \quad$ internal liquid inlet temperature $\left[{ }^{\circ} \mathrm{C}\right]$

$T^{*} \quad$ internal fluid temperature profile $\left[{ }^{\circ} \mathrm{C}\right]$

$T_{e} \quad$ external fluid temperature $\left[{ }^{\circ} \mathrm{C}\right]$ 


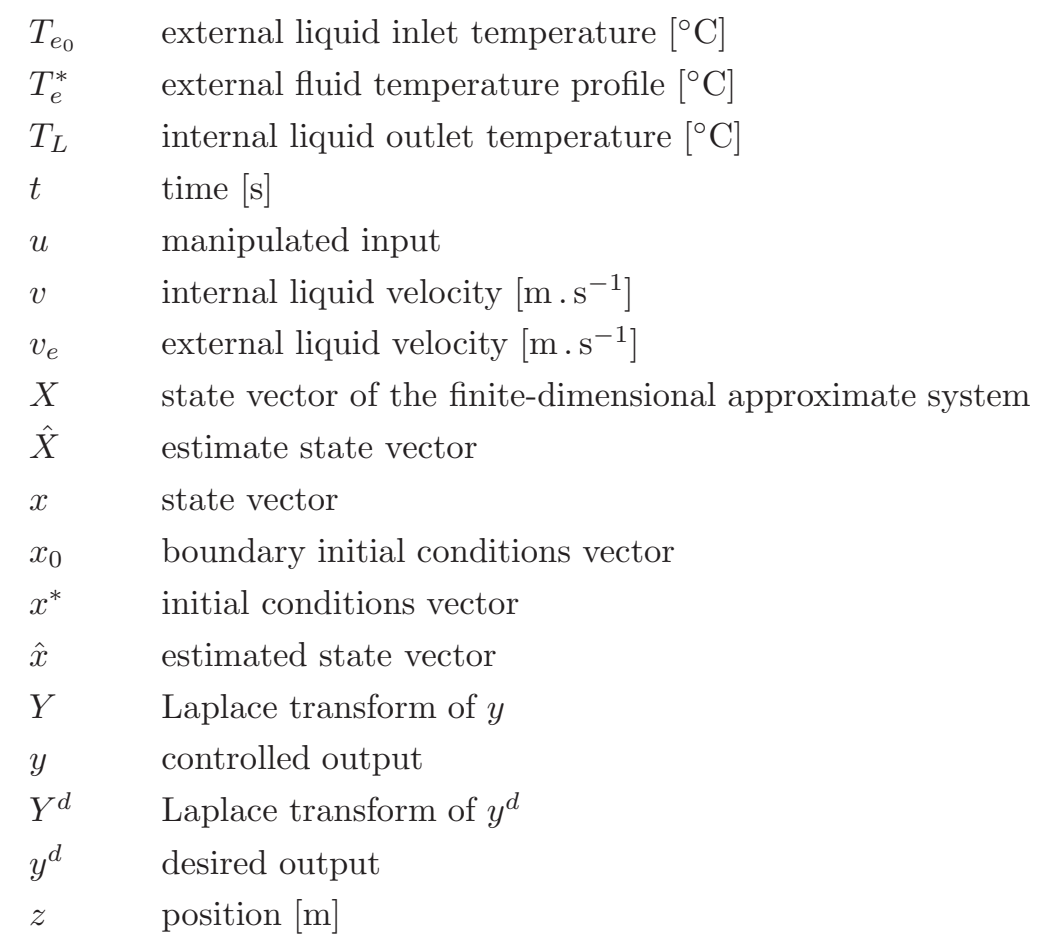

Greek symbols

$\beta_{1} \quad$ flow rate controller parameter [s]

$\beta_{2} \quad$ flow rate controller parameter $\left[\mathrm{s}^{2}\right]$

$\Delta z, \Delta L$ distance between two adjacent discretization points [m]

$\epsilon \quad$ small length increment [m]

$\eta \quad$ measurement noise

$\nu \quad$ external input

$\rho \quad$ internal liquid density $\left[\mathrm{kg} \cdot \mathrm{m}^{-3}\right]$

$\rho_{e} \quad$ external liquid density $\left[\mathrm{kg} \cdot \mathrm{m}^{-3}\right]$

$\tau \quad$ time constant $[\mathrm{s}]$

$\tau_{I} \quad$ integral time constant of the PI controller [s]

$\xi \quad$ variable of integration

$\zeta \quad$ process noise

Subscripts and superscripts

$i, j \quad$ positions

$f \quad$ Kalman filter

$T \quad$ transpose

\section{Abbreviations}

PI proportional integral controller

ODE ordinary differential equation

PDE partial differential equation

LPS lumped parameter system

DPS distributed parameter system

ITAE integral of time multiplied by the absolute value of error 


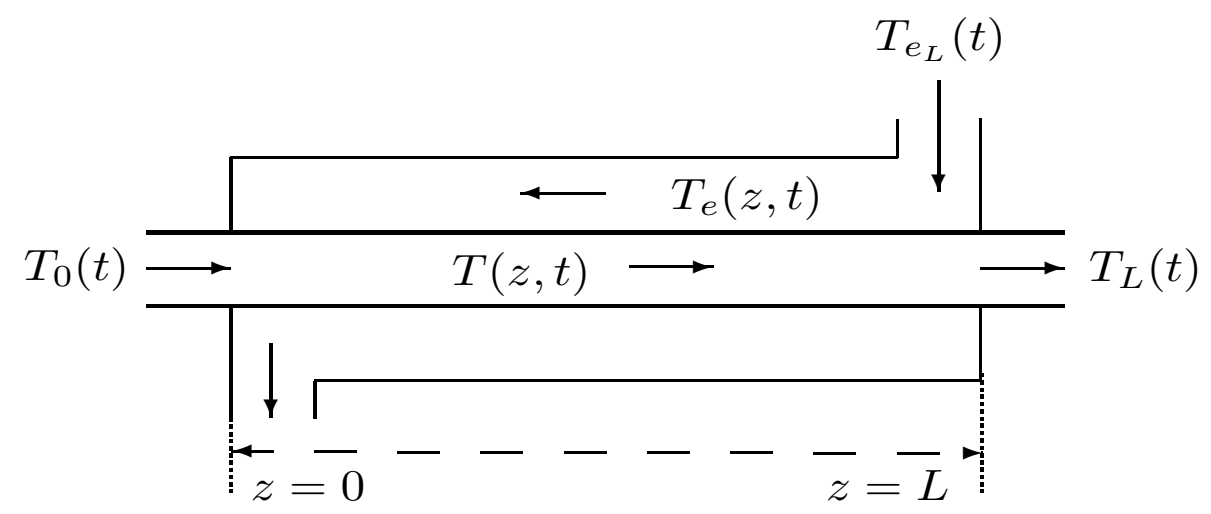

FiguRE 1 - Counter-current heat exchanger 
Input-Output system

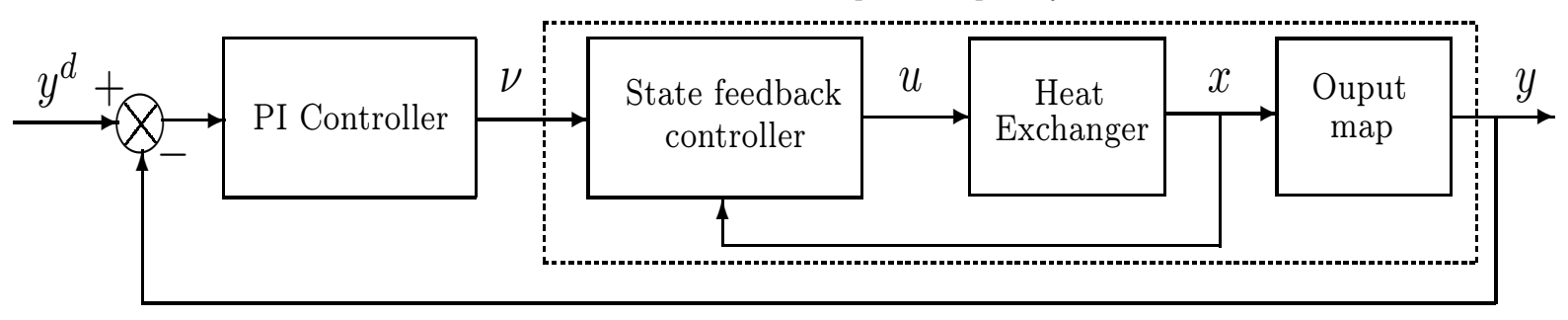

FiguRE 2 - Geometric control strategy of a counter-current heat exchanger 


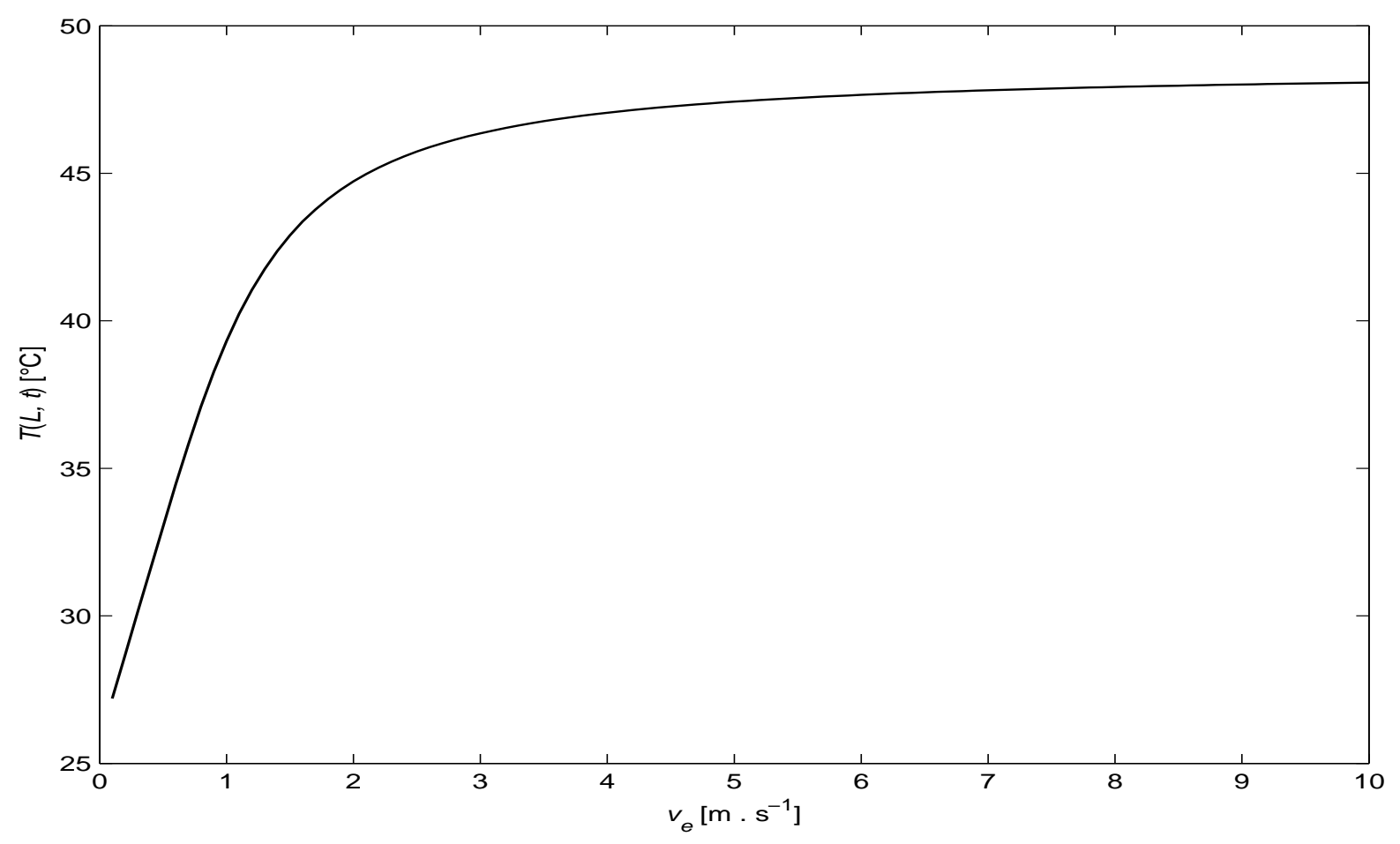

FiguRE 3 - Desired temperature at the outlet of the inner tube with respect to the velocity of the outer fluid in case of external flow rate control $\left(T_{e}(L)=50^{\circ} \mathrm{C}\right)$ 


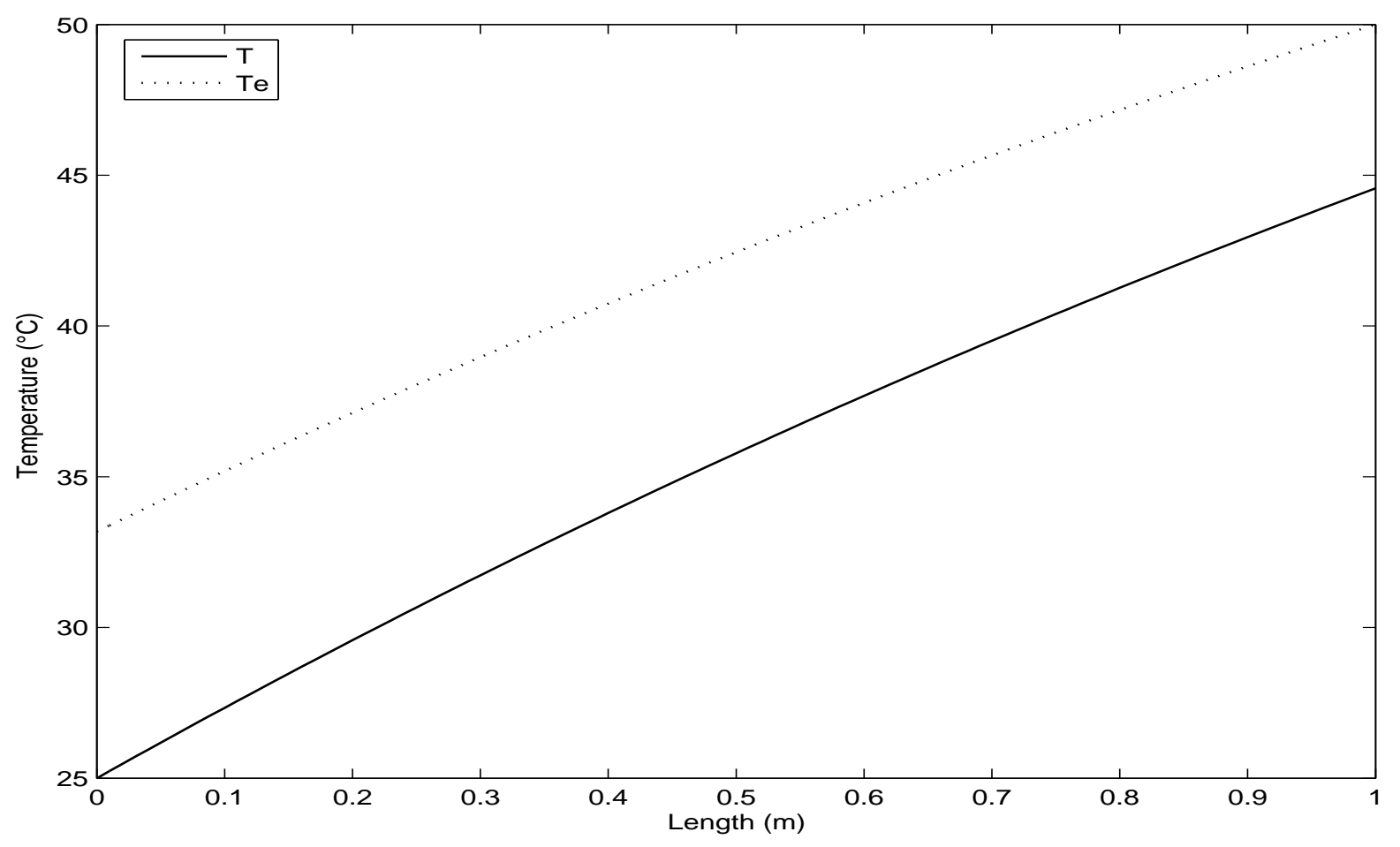

FIGURE 4 - Profiles of the temperatures of the internal and external fluids at steady state 

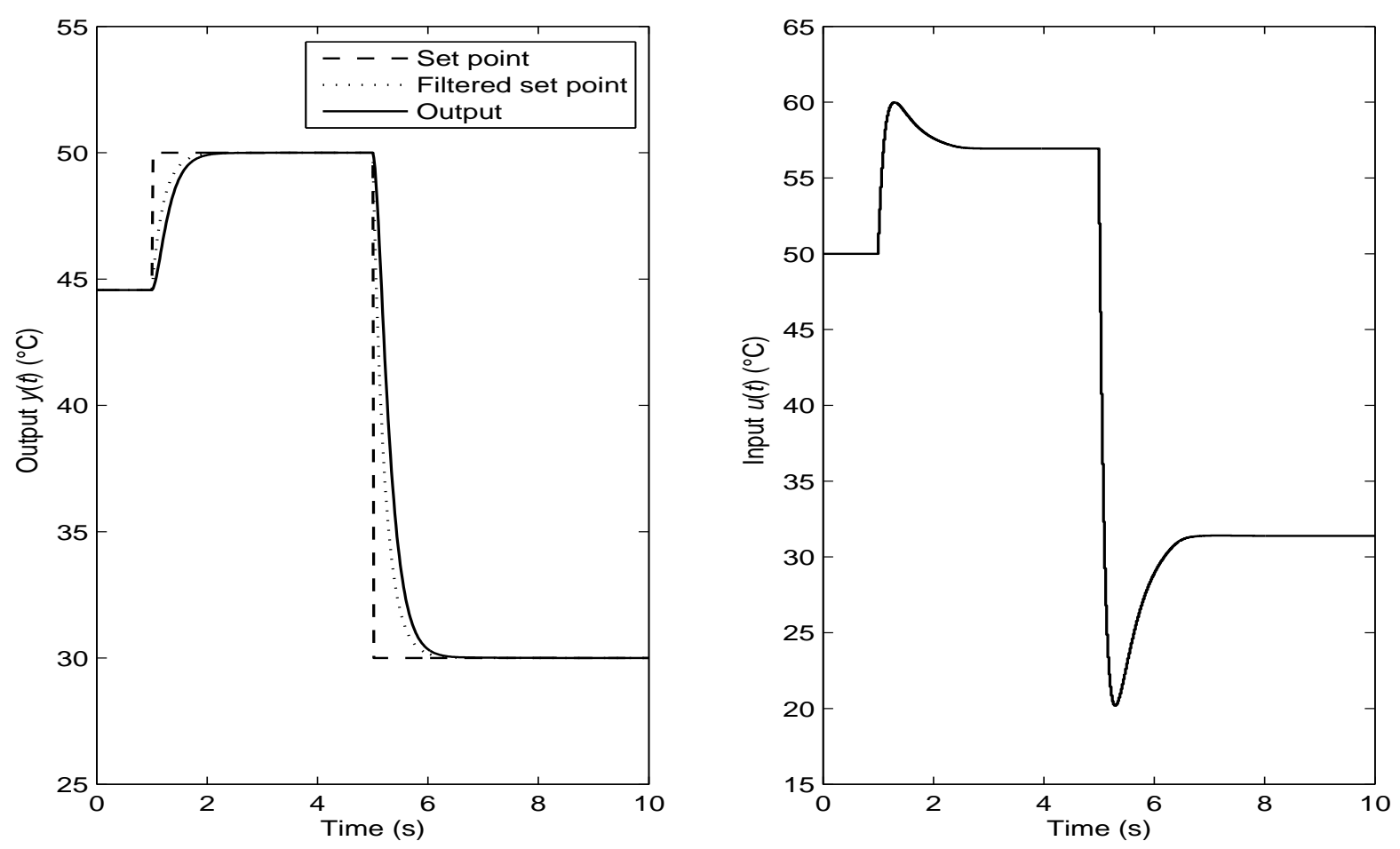

Figure 5 - Case of set point step. Left : Controlled output and set point. Right : Manipulated input 

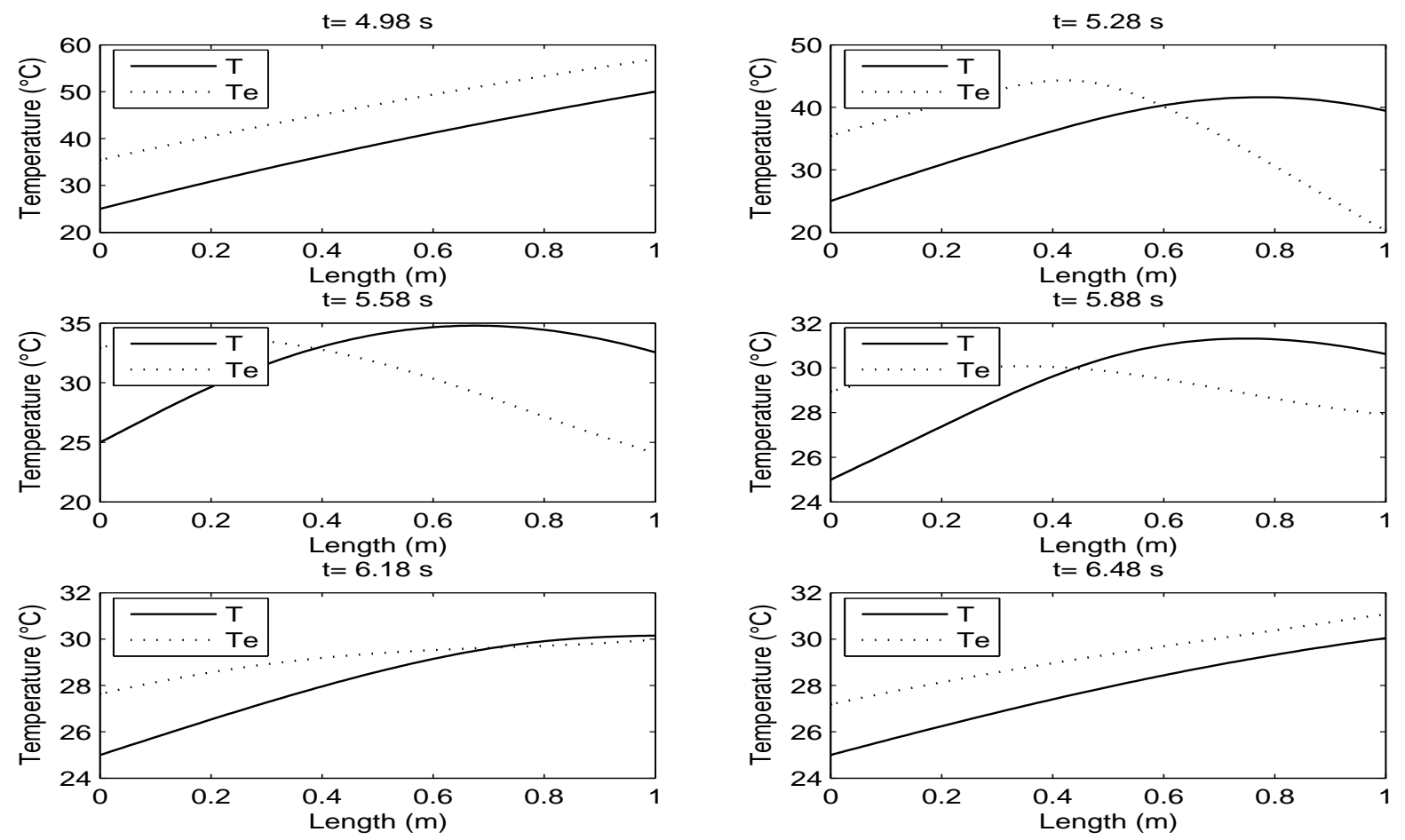

FiguRE 6 - Temperature profiles and crossover effect in the case of negative set point variation of $-20^{\circ} \mathrm{C}$ at $t=5 \mathrm{~s}$. 

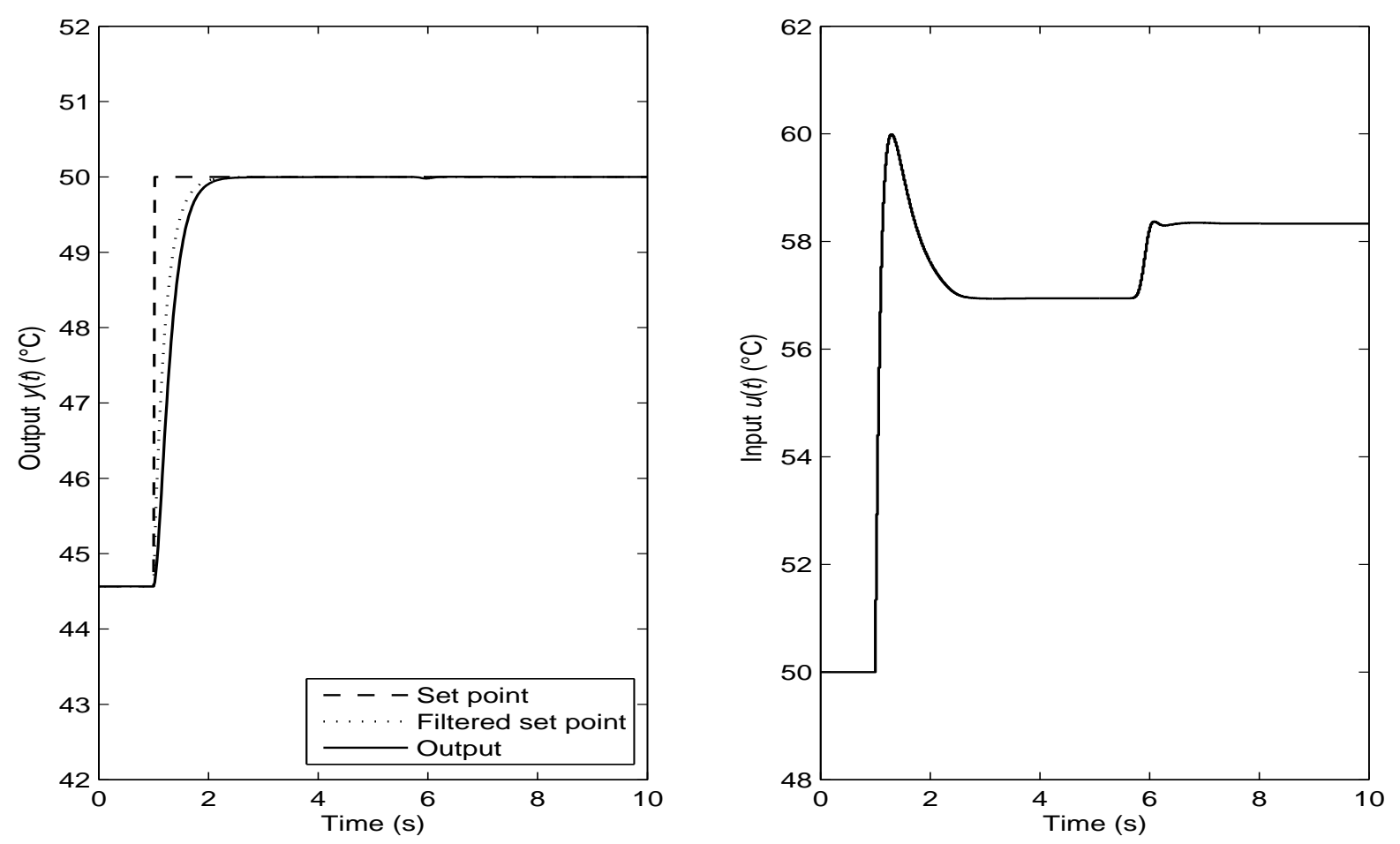

FiguRE 7 - Case of disturbance rejection. Left : Controlled output. Right : Manipulated input 


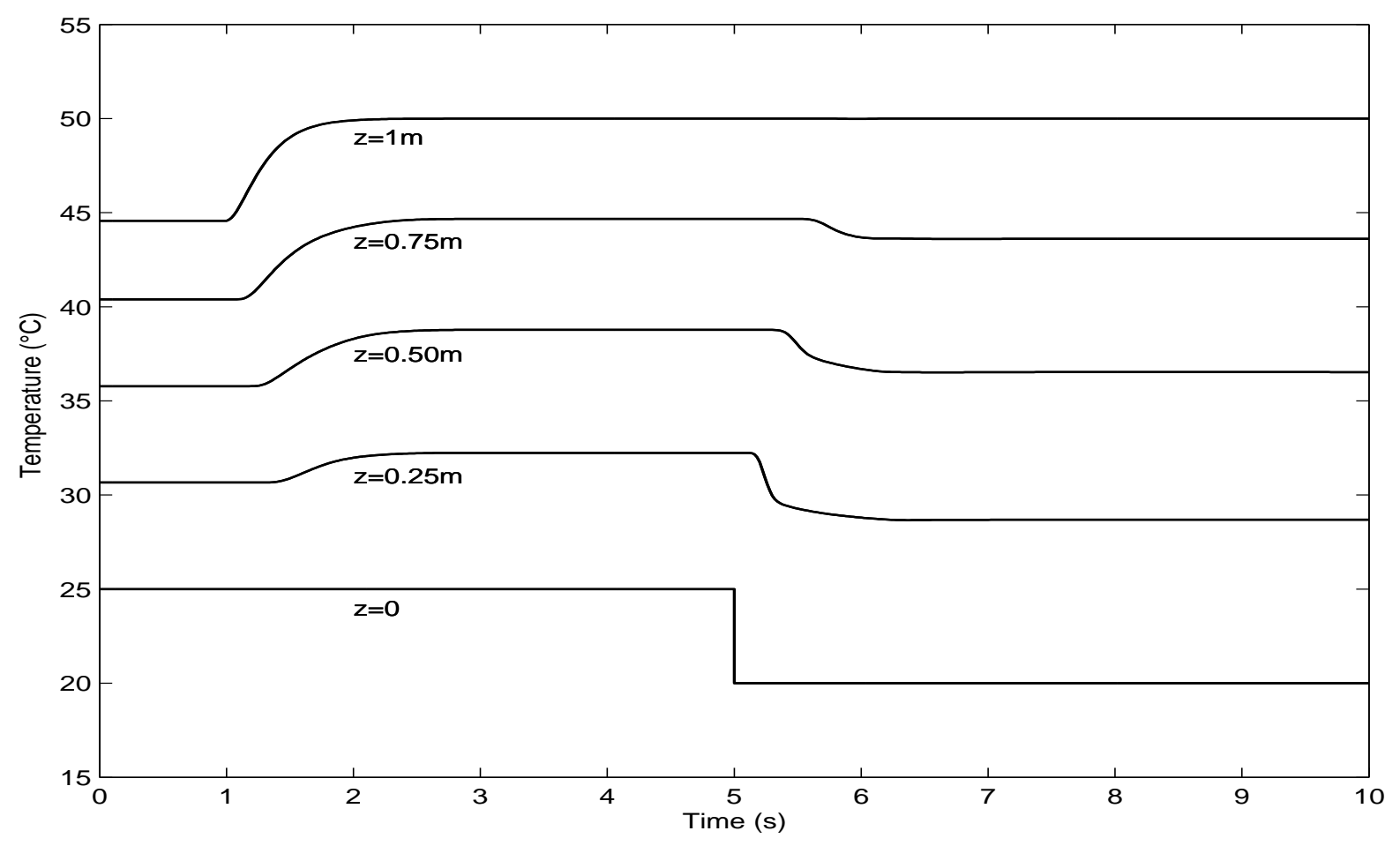

FiguRE 8 - Variation of temperature at different locations along the heat exchanger in the case of disturbance rejection (the disturbance is the internal fluid temperature at $z=0$ ) 


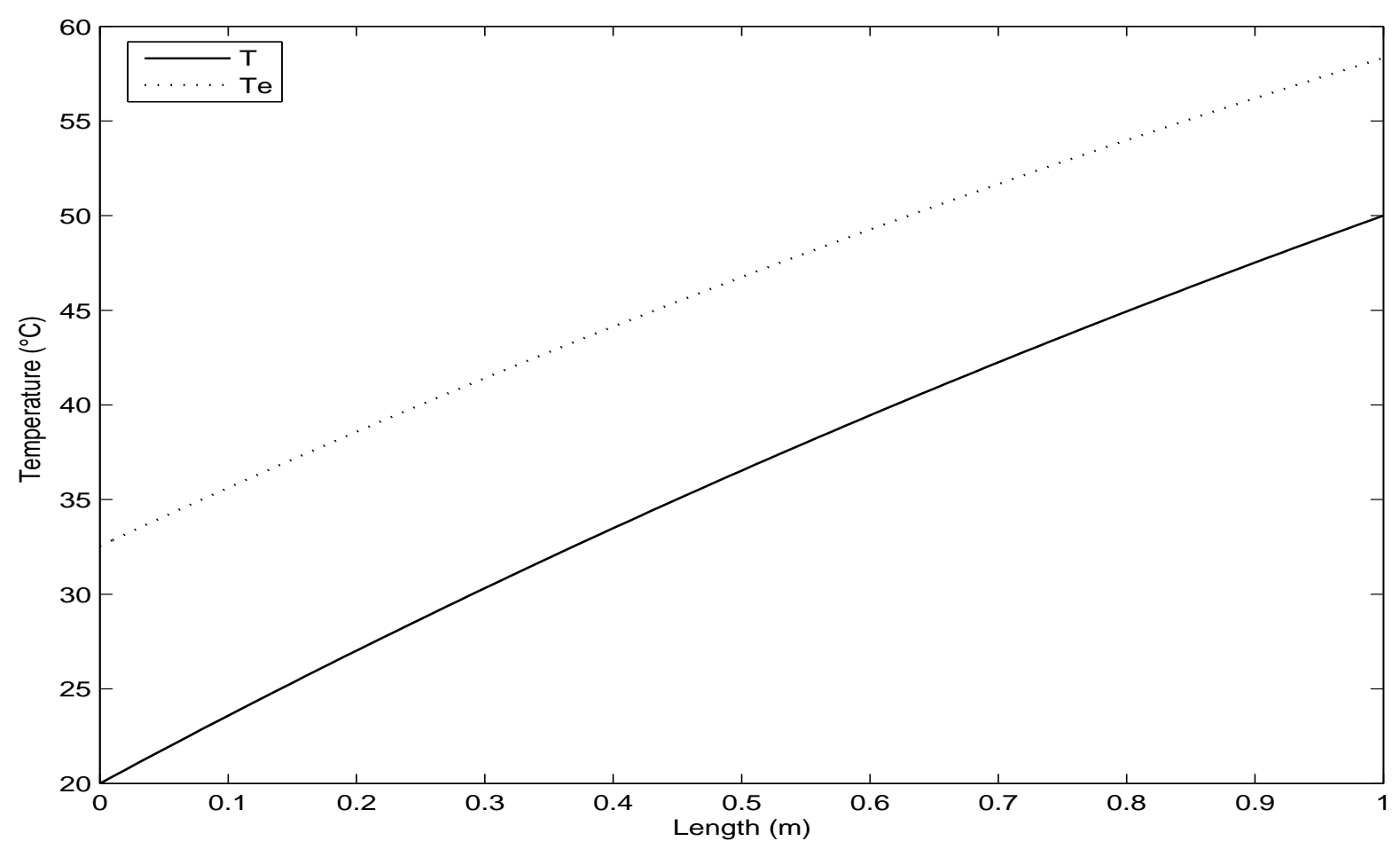

FIGURE 9 - Spatial profiles of temperatures at time $t=10 \mathrm{~s}$ in the case of disturbance rejection 

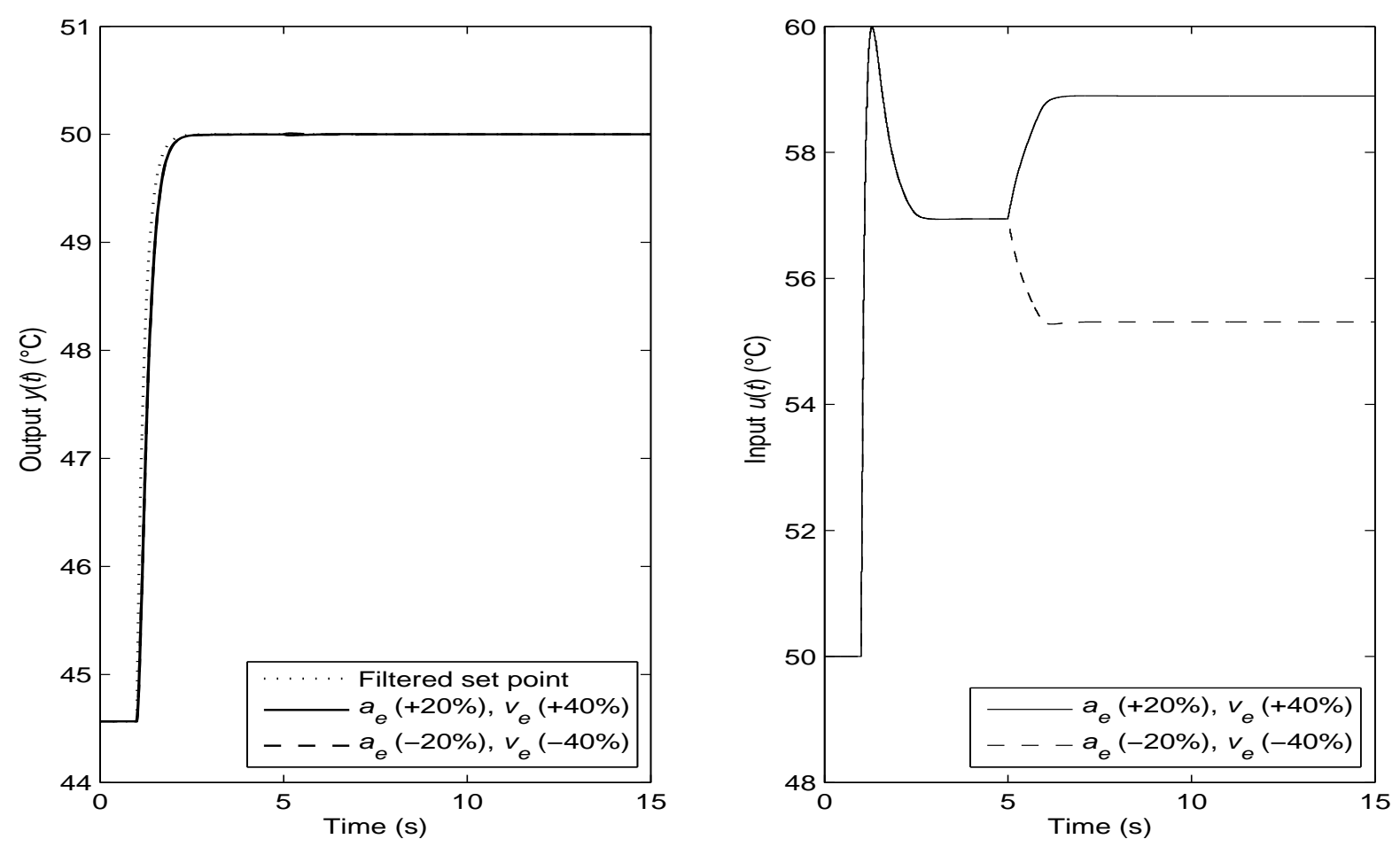

Figure 10 - Case of parameter change for the external fluid. Left : Controlled output and set point. Right : Manipulated input 

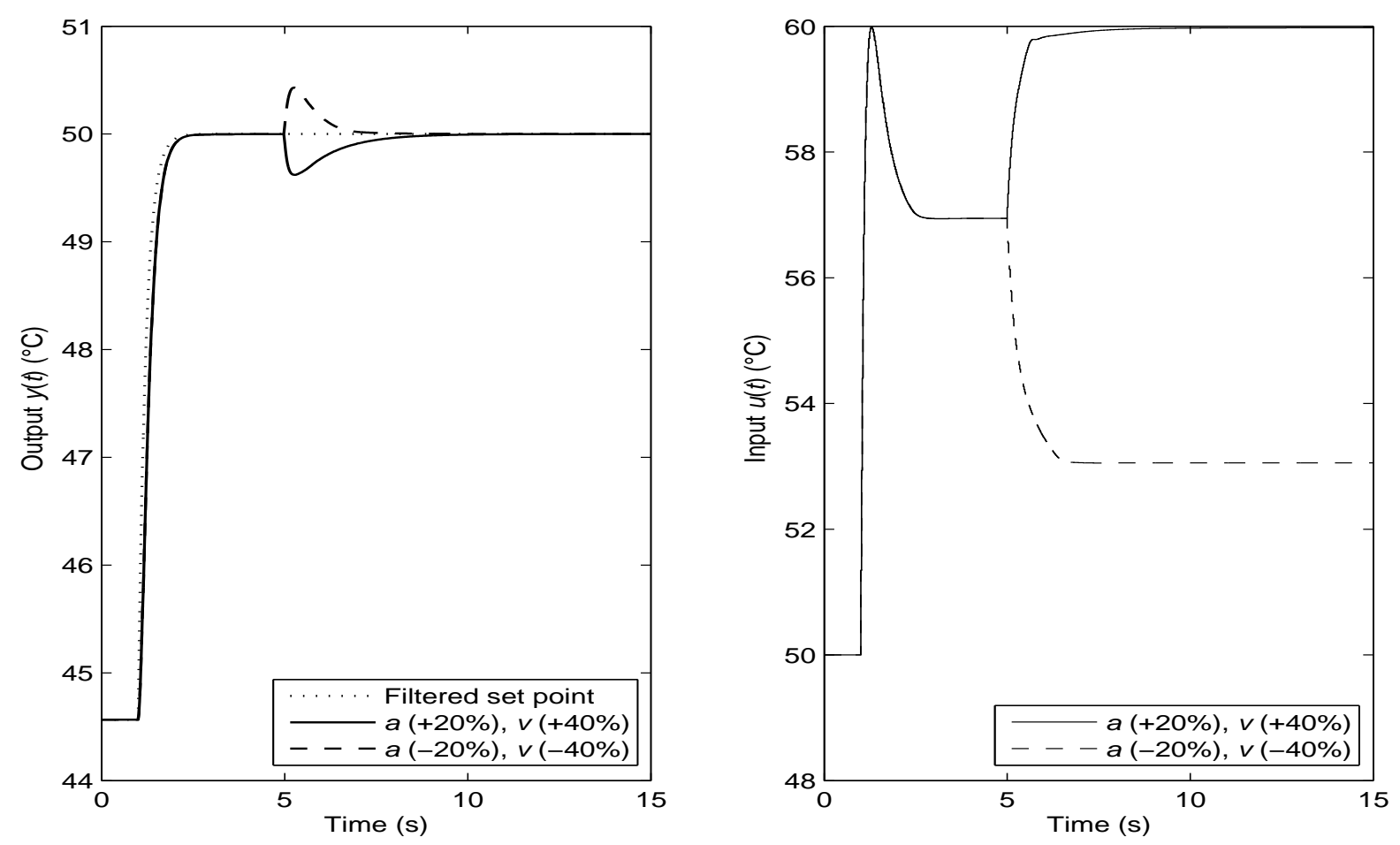

FiguRE 11 - Case of parameter change for the internal fluid with a constant set point. Left : Controlled output and set point. Right : Manipulated input 

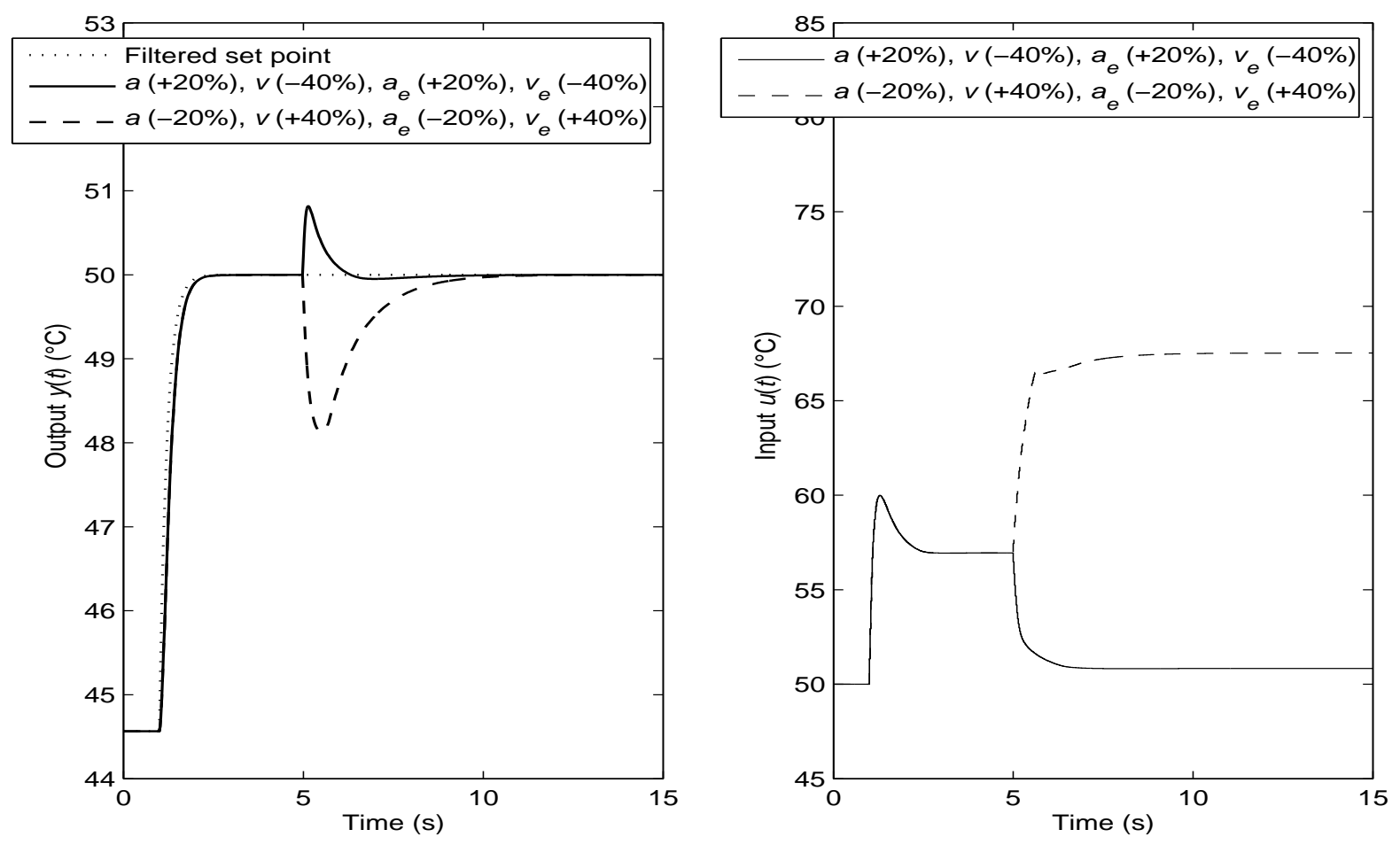

Figure 12 - Case of parameter change for the two fluids with a constant set point. Left : Controlled output and set point. Right : Manipulated input 

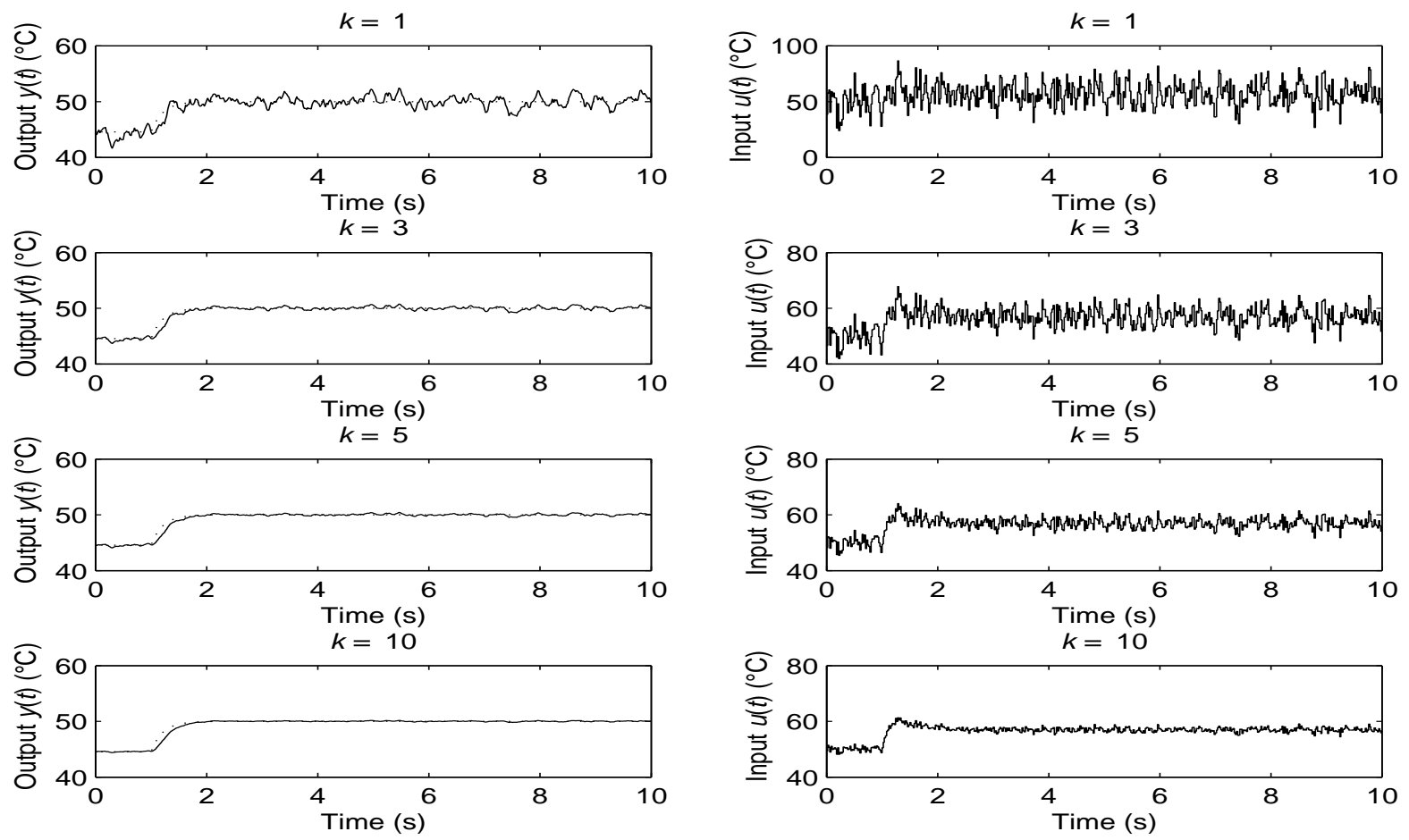

FIGURE 13 - Influence of the location of the sensor that measures the temperature at position $z=$ $L-\Delta L=L-k \Delta z$. Left : Output response. Right : manipulated input 

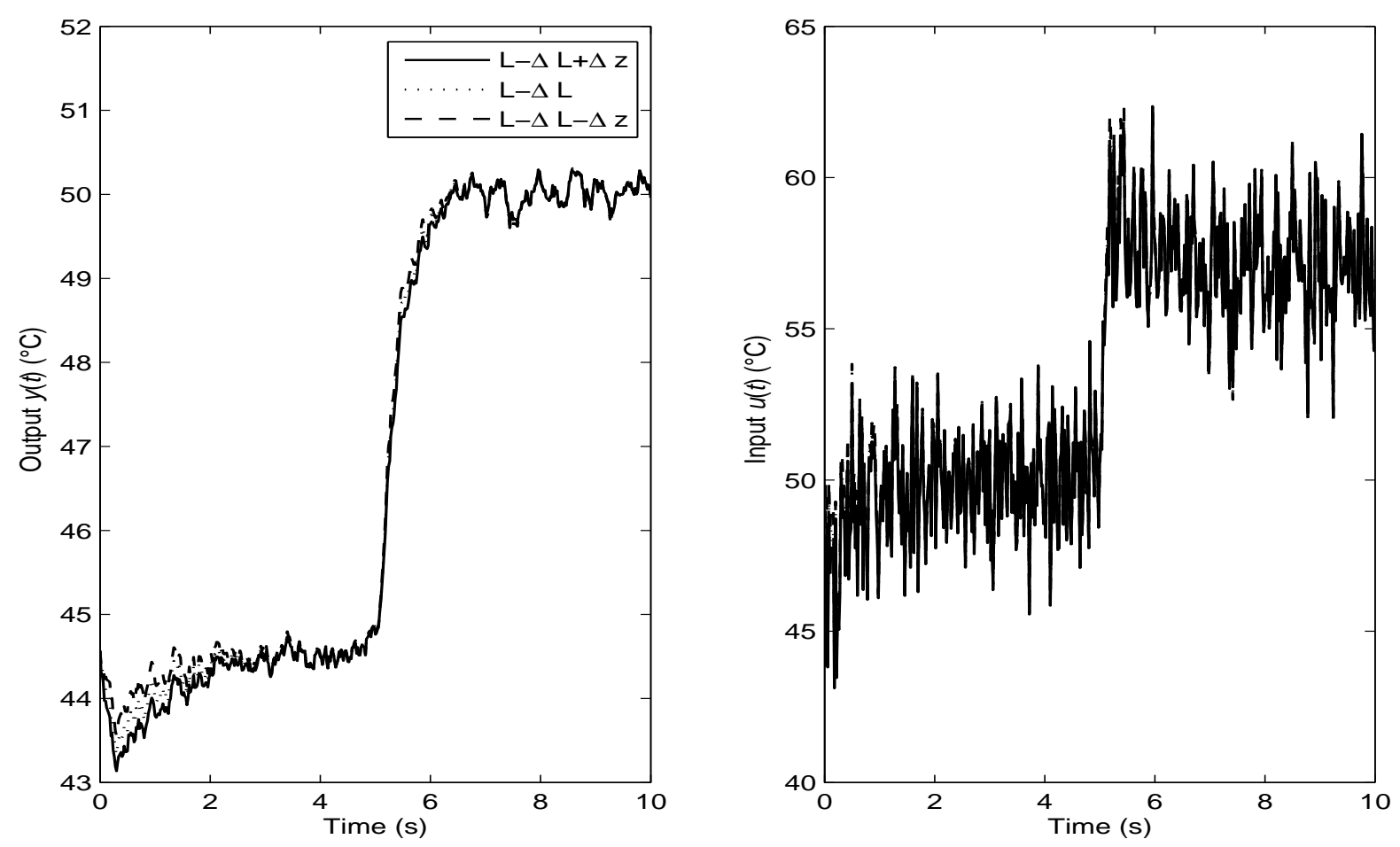

FIGURE 14 - Influence of the error location of the sensor that measures the temperature at position $z=L-\Delta L$. Left : Output response. Right : manipulated input 


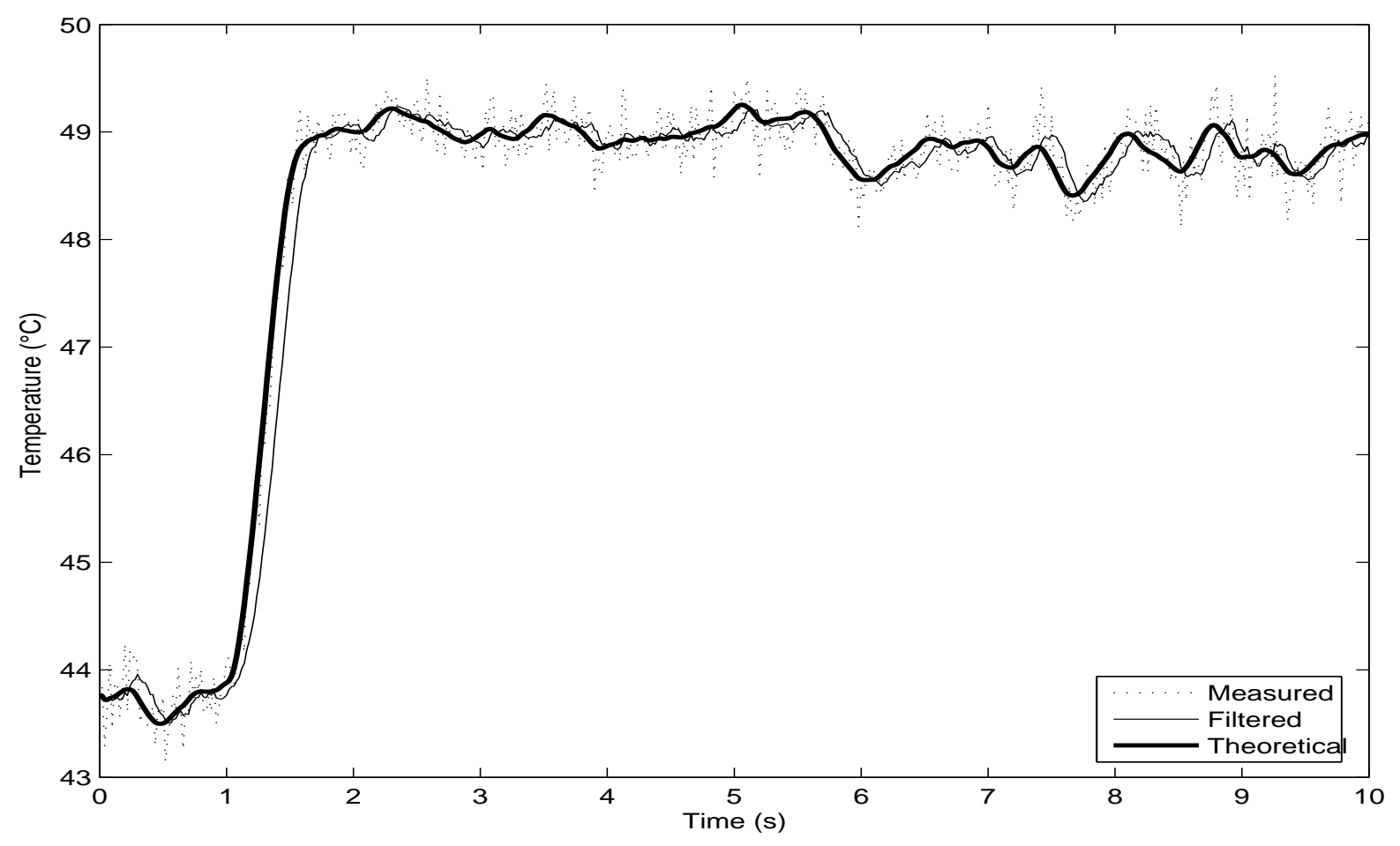

FIGURE 15 - Filtering of measurements : temperature at location $z=L-\Delta L(\Delta L=5 \Delta z)$ 


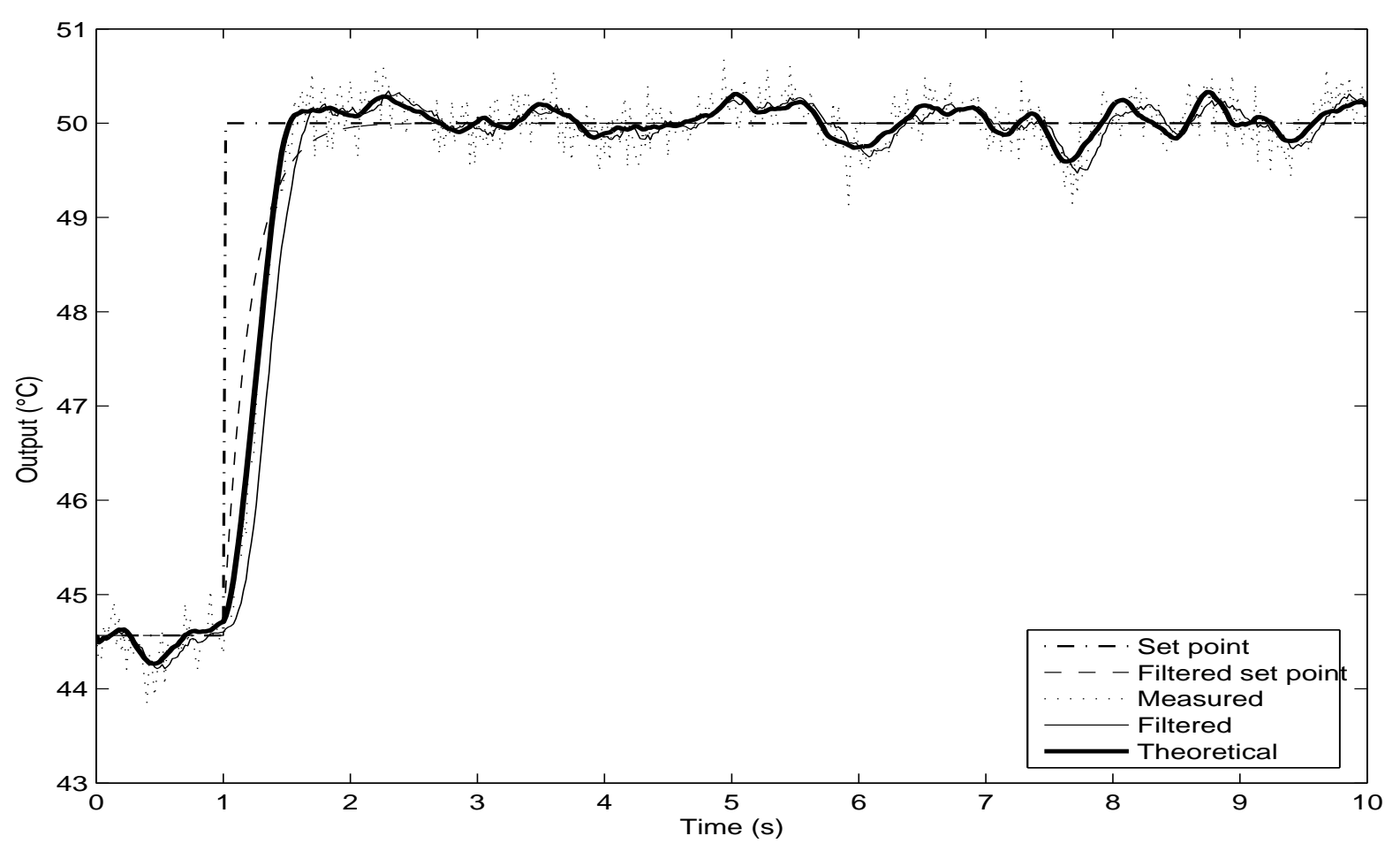

FiguRE 16 - Filtering of measurements : temperature at location $z=L(\Delta L=5 \Delta z)$ 


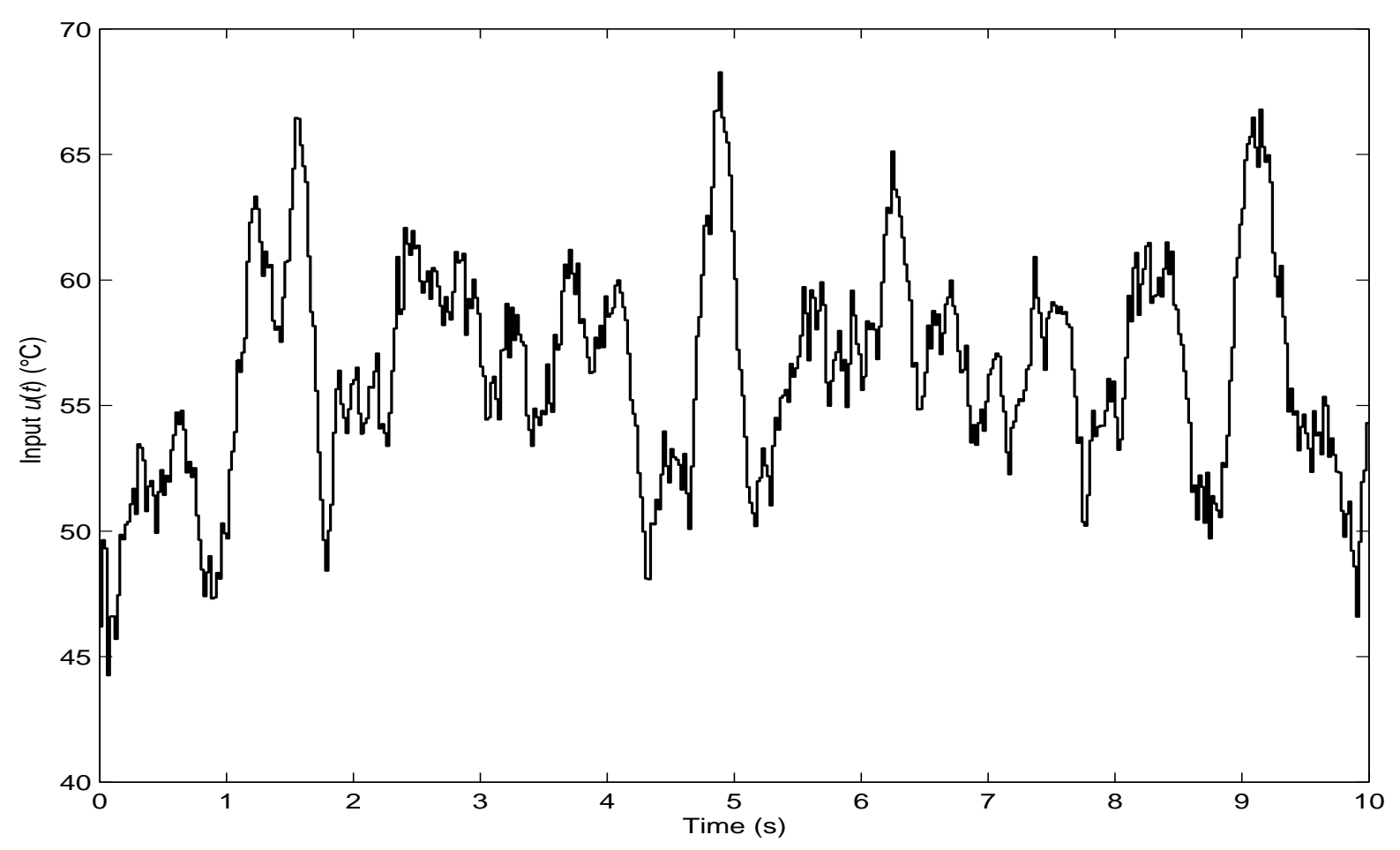

Figure 17 - Manipulated input in the case of measurement noise and state estimation by simple filtering $(\Delta L=5 \Delta z)$ 


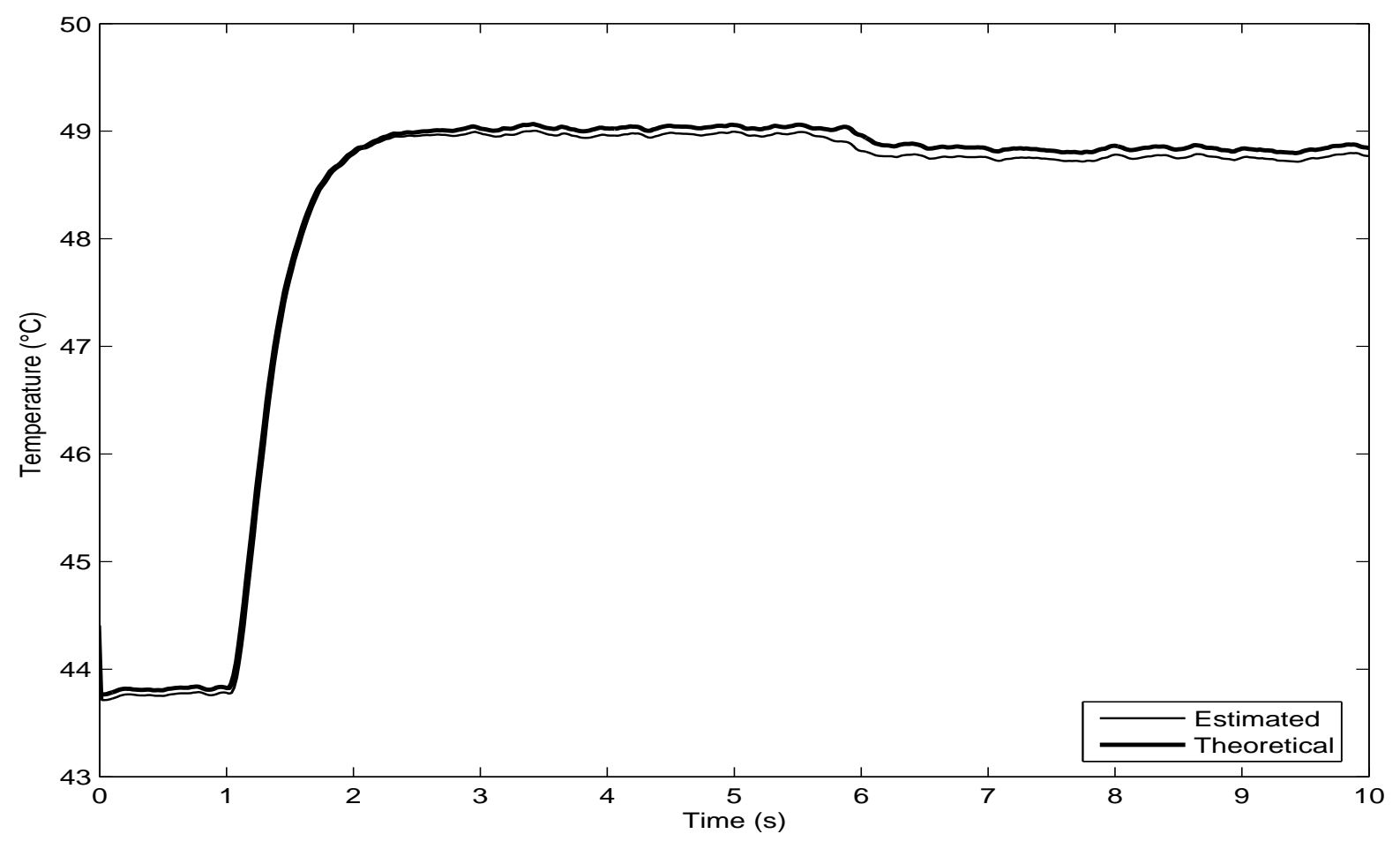

FIGURE 18 - Estimation of the temperature at location $z=L-\Delta L$ by Kalman filter $(\Delta L=5 \Delta z)$ 


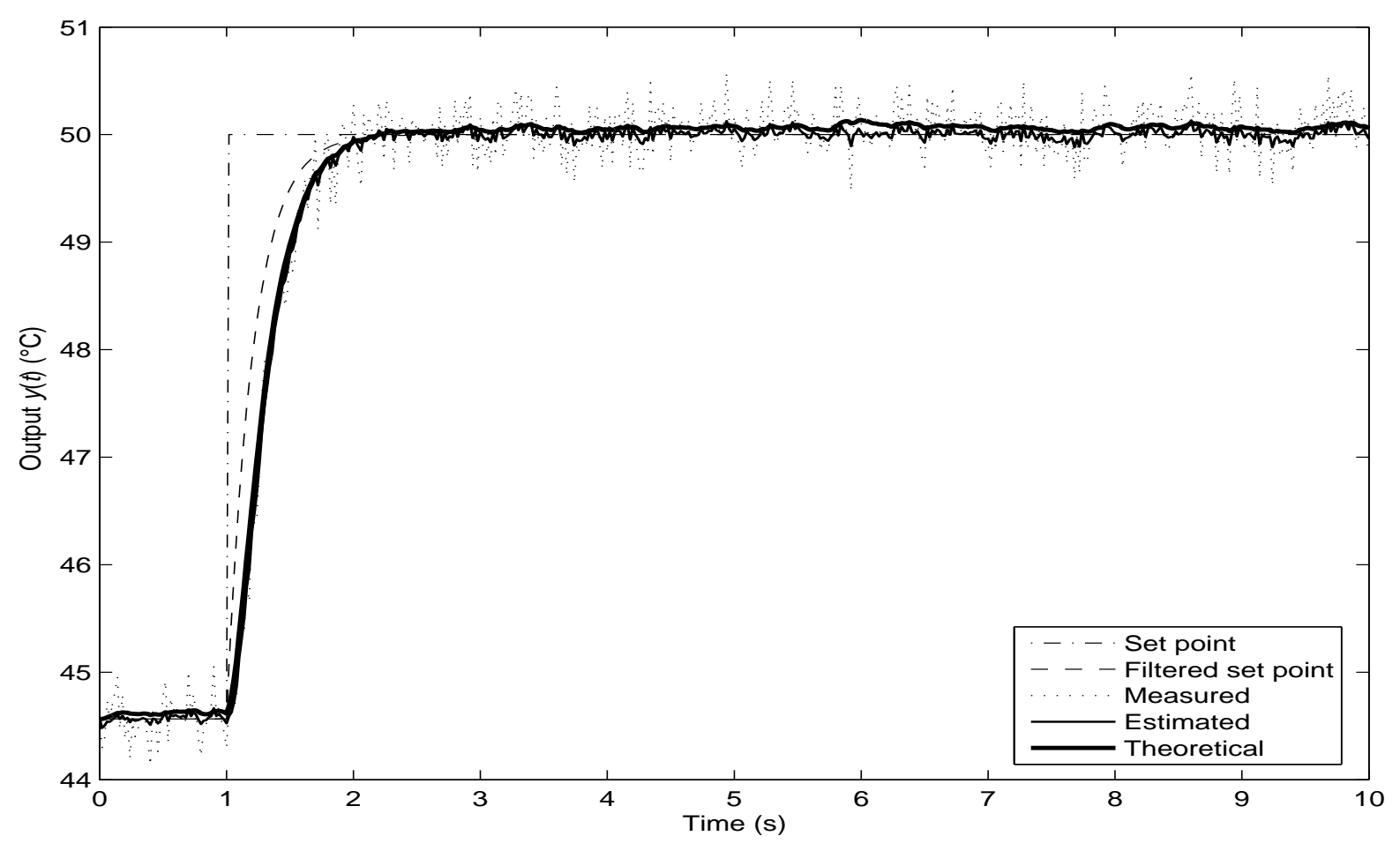

FIGURE 19 - Estimation of the temperature at location $z=L(\Delta L=5 \Delta z)$ by Kalman filter 


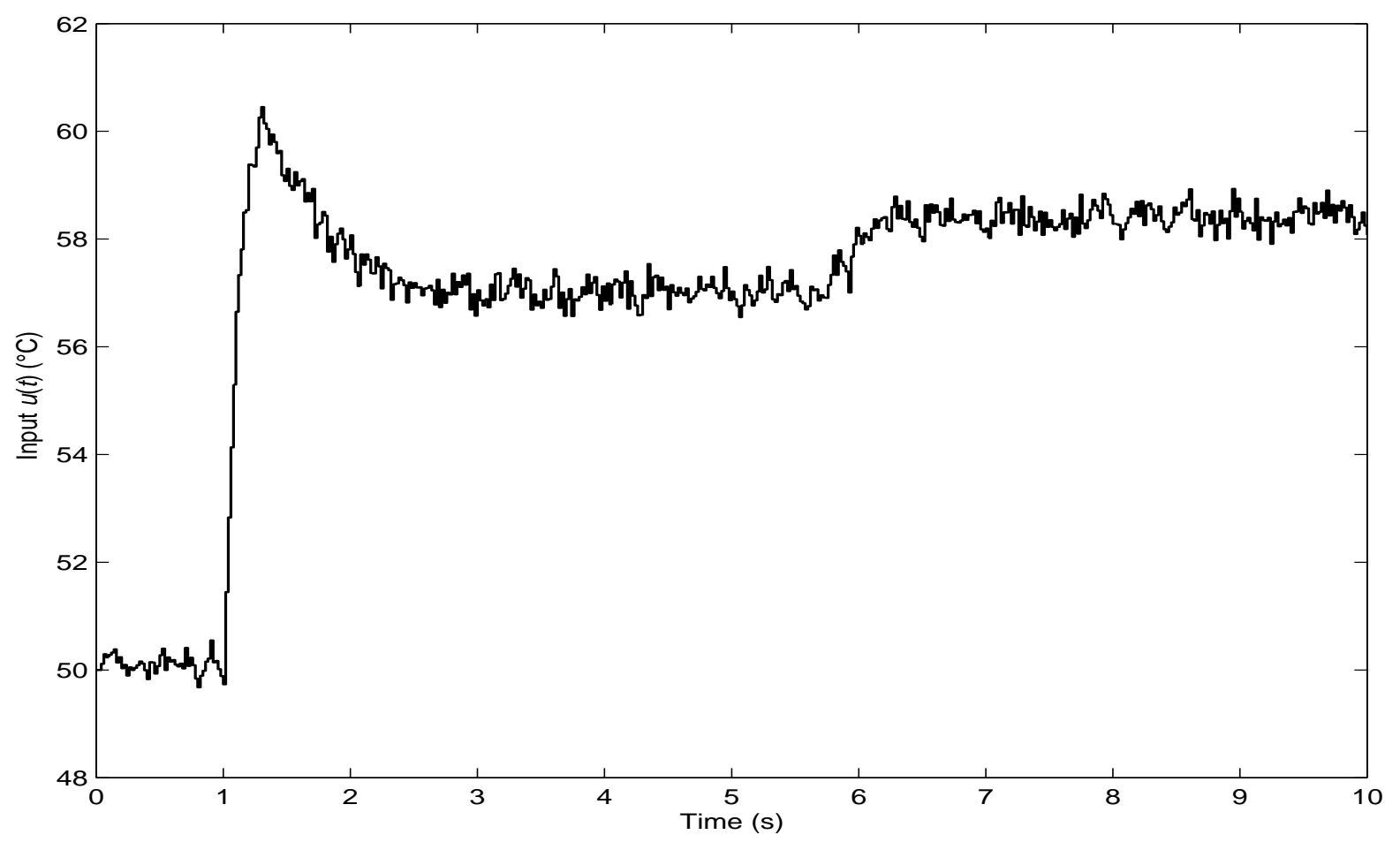

FIGURE 20 - Manipulated input in the case of state estimation by Kalman filter $(\Delta L=5 \Delta z)$ 


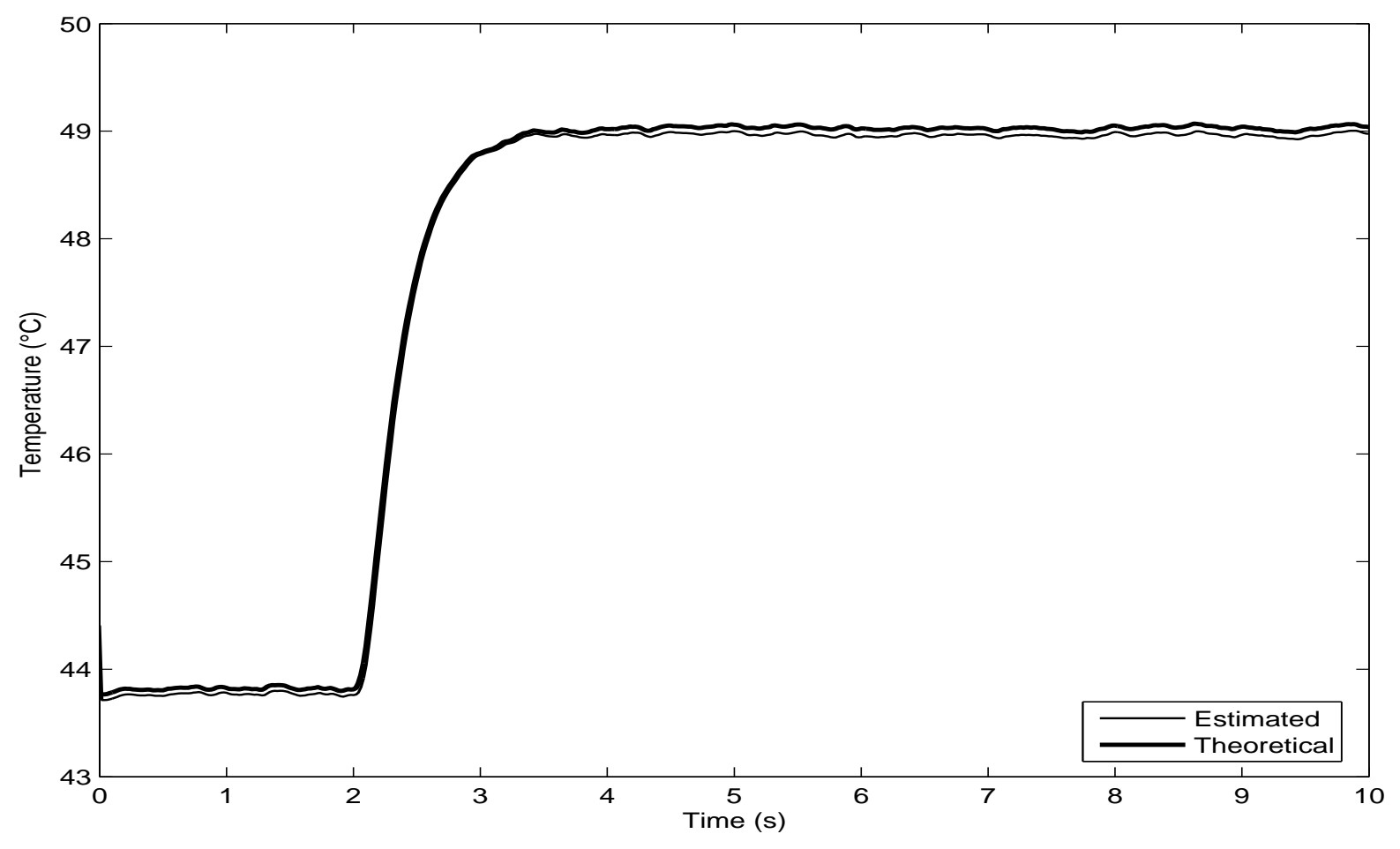

FIGURE 21 - Estimation of the temperature at location $z=L-\Delta L(\Delta L=5 \Delta z)$ for parameter change : $a(-25 \%), v(-25 \%), a_{e}(+25 \%), v_{e}(+25 \%)$ 


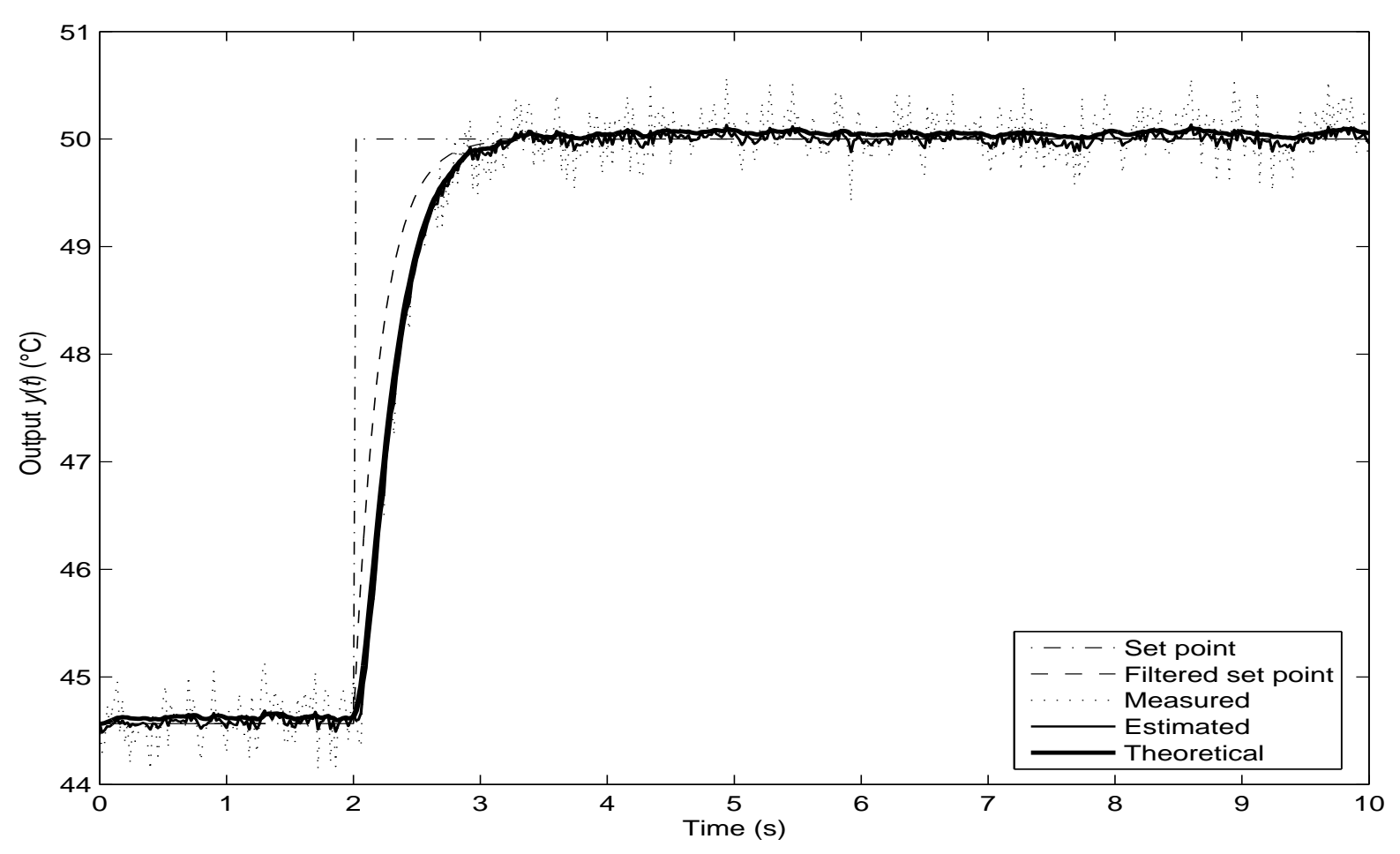

FiguRE 22 - Estimation of the temperature at location $z=L(\Delta L=5 \Delta z)$ for parameter change : $a(-25 \%), v(-25 \%), a_{e}(+25 \%), v_{e}(+25 \%)$ 


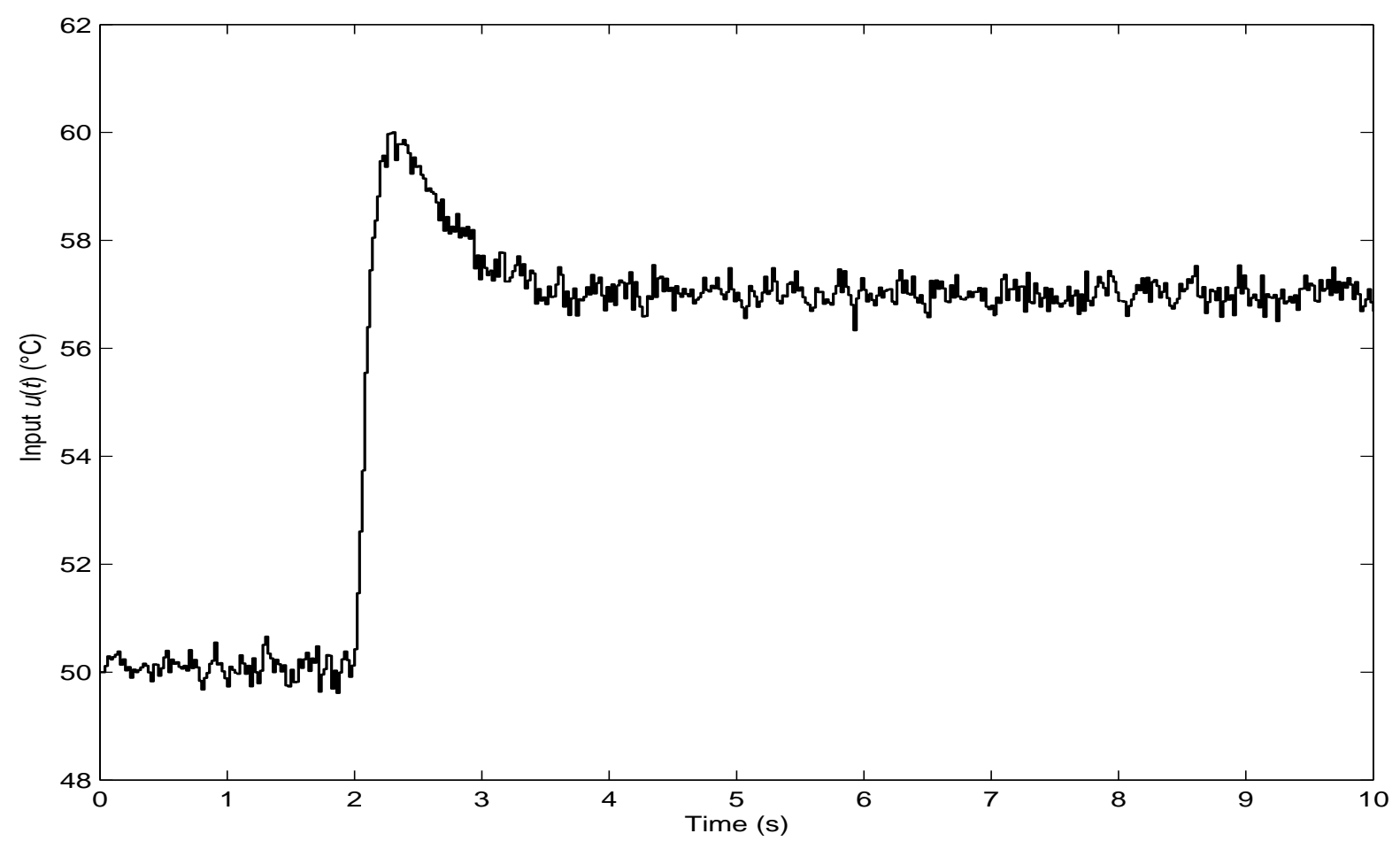

FIGURE 23 - Manipulated input in the case of state estimation with observer $(\Delta L=5 \Delta z)$ for parameter change : $a(-25 \%), v(-25 \%), a_{e}(+25 \%), v_{e}(+25 \%)$ 

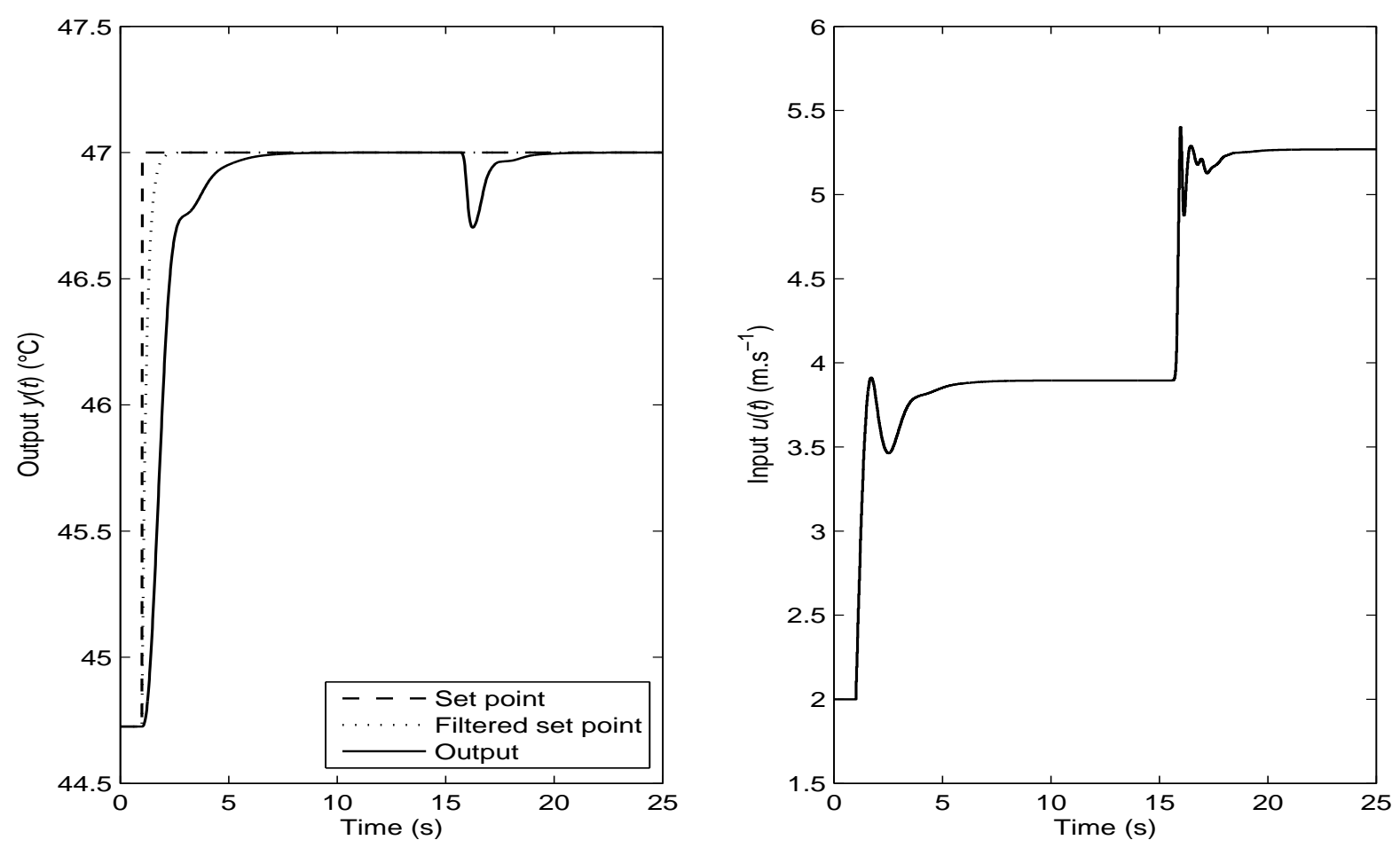

FIGURE 24 - Manipulated of the flow rate of the external fluid. Left : Output response. Right : manipulated input 